TRANSACTIONS OF THE

AMERICAN MATHEMATICAL SOCIETY

Volume 348, Number 11, November 1996

\title{
PROJECTIVELY BOUNDED FRÉCHET MEASURES
}

\author{
RON C. BLEI
}

\begin{abstract}
A scalar valued set function on a Cartesian product of $\sigma$-algebras is a Fréchet measure if it is a scalar measure independently in each coordinate. A basic question is considered: is it possible to construct products of Fréchet measures that are analogous to product measures in the classical theory?

A Fréchet measure is said to be projectively bounded if it satisfies a Grothendieck type inequality. It is shown that feasibility of products of Fréchet measures is linked to the projective boundedness property. All Fréchet measures in a two dimensional framework are projectively bounded, while there exist Fréchet measures in dimensions greater than two that are projectively unbounded. A basic problem is considered: when is a Fréchet measure projectively bounded? Some characterizations are stated. Applications to harmonic and stochastic analysis are given.
\end{abstract}

\section{INTRODUCTION}

A scalar valued set function on a Cartesian product of algebras of sets is said to be a Fréchet measure if it is a scalar measure independently in each coordinate. A basic question is whether it is possible to construct products of Fréchet measures that are analogous to product measures in the usual framework. Issues concerning this question in the multidimensional setting, naturally arising, for example, in contexts of harmonic and stochastic analysis, are fundamentally different and indeed more challenging than corresponding issues in the classical one-dimensional setting.

A Fréchet measure is said to be projectively bounded if it satisfies a Grothendiecktype inequality. In this paper I establish a connection between existence of products and the projective boundedness property, and then consider the problem: when is a Fréchet measure projectively bounded?

The paper is organized into 11 sections:

1. Introduction.

2. Preliminaries.

3. $\mu$ is projectively bounded if and only if $\mu \times \nu \in \mathcal{F}_{n}$ for all $\nu \in \mathcal{F}_{n}$.

4. Harmonic analysis of $\mathcal{F}$-measures.

5. Every $\mu \in \mathcal{F}_{2}$ is projectively bounded.

6. There exist projectively unbounded $\mathcal{F}_{3}$-measures.

7. A characterization of projective boundedness.

8. Another characterization.

9. Applications to stochastic analysis.

Received by the editors April 28, 1995.

1991 Mathematics Subject Classification. Primary 28B99, 46G10, 46A32; Secondary 43A05, $60 \mathrm{G} 05$.

(C)1996 American Mathematical Society 
10. Projective boundedness, $L^{2}$-factorizability, complete boundedness, and convolvability.

11. Appendix.

Definition 1.1. Let $\mathcal{X}_{1}, \ldots, \mathcal{X}_{n}$ be sets, and let $\mathcal{C}_{1}, \ldots, \mathcal{C}_{n}$ be algebras of sets in $\mathcal{X}_{1}, \ldots, \mathcal{X}_{n}$, respectively. A scalar valued set function on $\mathcal{C}_{1} \times \cdots \times \mathcal{C}_{n}$ is an $\mathcal{F}_{n^{-}}$ measure if it is a scalar measure independently in each coordinate. The space of $\mathcal{F}_{n}$-measures on $\mathcal{C}_{1} \times \cdots \times \mathcal{C}_{n}$ is denoted by $\mathcal{F}_{n}\left(\mathcal{C}_{1} \times \cdots \times \mathcal{C}_{n}\right)$, or by $\mathcal{F}_{n}\left(\cdot \times \mathcal{X}_{i} \times \cdot \cdot\right)$ if $\mathcal{C}_{i}$ is the power set of $\mathcal{X}_{i}$. If $\mathcal{C}_{1}, \ldots, \mathcal{C}_{n}$ are arbitrary or understood from the context, then $\mathcal{F}_{n}\left(\mathcal{C}_{1} \times \cdots \times \mathcal{C}_{n}\right)$ is denoted by $\mathcal{F}_{n}$. (A norm on $\mathcal{F}_{n}$, extending the total variation norm in the case $n=1$, is defined in the next section; see (2.5)).

$\mathcal{F}_{2}$-measures appeared implicitly in Fréchet's characterization of bounded bilinear functionals on $C([0,1])[\mathrm{F}]$. These bilinear functionals were dubbed bimeasures by Morse and Transue (e.g., [M]), but a Riesz representation-type theorem, actually identifying bimeasures as bona fide set functions on the two-fold product of the respective Borel fields, was stated and proved first by Ylinen [Y1, Theorem 6.9]. In multidimensional frameworks, be they topological or measurable, analogous multilinear functionals or scalar valued set functions on products of $\sigma$-algebras have been called sometimes multimeasures and sometimes polymeasures (e.g., [D], [GY]). I use the term $\mathcal{F}_{n}$-measure in a general context of multidimensional measure theory.

Let $\left(\mathcal{X}_{i}, \mathfrak{A}_{i}\right),\left(\mathcal{Y}_{i}, \mathfrak{B}_{i}\right)(i=1, \ldots, n)$ be measurable spaces. For

$$
\mu \in \mathcal{F}_{n}\left(\mathfrak{A}_{1} \times \cdots \times \mathfrak{A}_{n}\right) \text { and } \quad \nu \in \mathcal{F}_{n}\left(\mathfrak{B}_{1} \times \cdots \times \mathfrak{B}_{n}\right),
$$

define

$$
\begin{aligned}
\mu \times \nu\left(\left(A_{1}, B_{1}\right), \ldots,\left(A_{n}, B_{n}\right)\right)=\mu\left(A_{1}, \ldots, A_{n}\right) \nu\left(B_{1}, \ldots, B_{n}\right), & \\
& \left(A_{1}, \ldots, A_{n}\right) \in \mathfrak{A}_{1} \times \cdots \times \mathfrak{A}_{n},\left(B_{1}, \ldots, B_{n}\right) \in \mathfrak{B}_{1} \times \cdots \times \mathfrak{B}_{n} .
\end{aligned}
$$

Question. Can $\mu \times \nu$ be extended to an $\mathcal{F}_{n}$-measure on $\sigma\left(\mathfrak{A}_{1} \times \mathfrak{B}_{1}\right)$ $\times \cdots \times \sigma\left(\mathfrak{A}_{n} \times \mathfrak{B}_{n}\right) ?(\sigma(\mathfrak{A} \times \mathfrak{B})$ denotes the $\sigma$-algebra generated by $\mathfrak{A} \times \mathfrak{B}$. $)$

Definition 1.2. Let $\left(\mathcal{X}_{1}, \mathfrak{A}_{1}\right), \ldots,\left(\mathcal{X}_{n}, \mathfrak{A}_{n}\right)$ be measurable spaces and let $\mu \in$ $\mathcal{F}_{n}\left(\mathfrak{A}_{1} \times \cdots \times \mathfrak{A}_{n}\right)$. For $F_{1} \subset \mathcal{L}^{\infty}\left(X_{1}\right), \ldots, F_{n} \subset \mathcal{L}^{\infty}\left(\mathcal{X}_{n}\right)$, write

$$
\phi_{\mu}\left(f_{1}, \ldots, f_{n}\right)=\int f_{1} \otimes \cdots \otimes f_{n} d \mu, \quad\left(f_{1}, \ldots, f_{n}\right) \in F_{1} \times \cdots \times F_{n},
$$

and then define

$$
\|\mu\|_{p b_{n}}=\sup \left\{\left\|\phi_{\mu}\right\|_{V_{n}\left(F_{1} \times \cdots \times F_{n}\right)}: F_{i} \subset \text { unit ball of } \mathcal{L}^{\infty}\left(\mathcal{X}_{i}\right),\left|F_{i}\right|<\infty, i \in[n]\right\} .
$$

If $\|\mu\|_{p b_{n}}<\infty$, then $\mu$ is said to be projectively bounded; if $\|\mu\|_{p b_{n}}=\infty$, then $\mu$ is said to be projectively unbounded. The class of projectively bounded $\mathcal{F}_{n}$-measures on $\mathfrak{A}_{1} \times \cdots \times \mathfrak{A}_{n}$ is denoted by $\mathcal{P} \mathcal{B F}_{n}=\mathcal{P} \mathcal{B F}_{n}\left(\mathfrak{A}_{1} \times \cdots \times \mathfrak{A}_{n}\right)$. (The integral on the right side of (1.2) and the projective tensor norm in (1.3) will be reviewed in the next section.)

To assert that $\mu \in \mathcal{P B \mathcal { F }}{ }_{n}$ is to say, in effect, that $\mu$ satisfies a Grothendiecktype inequality. The linear space $\mathcal{P B \mathcal { F }} \mathcal{F}_{n}$ equipped with the norm $\|\cdot\|_{p b_{n}}$ is a Banach space. The canonical imbedding $\mathcal{P} \mathcal{B F}_{n} \hookrightarrow \mathcal{F}_{n}$ is norm continuous (cf. (2.5)), and it is a surjection if and only if $n \leq 2$ (see sections 5, 6, and 10i). The 
following theorem, proved in section 3, is a link between construction of products and projective boundedness:

Theorem 1.3. Let $\left(\mathcal{X}_{1}, \mathfrak{A}_{1}\right), \ldots,\left(\mathcal{X}_{n}, \mathfrak{A}_{n}\right),\left(\mathcal{Y}_{n}, \mathfrak{B}_{1}\right), \ldots,\left(\mathcal{Y}_{n}, \mathfrak{B}_{n}\right)$ be measurable spaces, and assume that each of the $\sigma$-algebras $\mathfrak{B}_{1}, \ldots, \mathfrak{B}_{n}$ is infinite. Let $\mu \in$ $\mathcal{F}_{n}\left(\mathfrak{A}_{1} \times \cdots \times \mathfrak{A}_{n}\right)$. Then, $\mu \in \mathcal{P} \mathcal{B} \mathcal{F}_{n}\left(\mathfrak{A}_{1} \times \cdots \times \mathfrak{A}_{n}\right)$ if and only if $\mu \times \nu$ determines an $\mathcal{F}_{n}$-measure on $\sigma\left(\mathfrak{A}_{1} \times \mathfrak{B}_{1}\right) \times \cdots \times \sigma\left(\mathfrak{A}_{n} \times \mathfrak{B}_{n}\right)$ for every $\nu \in \mathcal{F}_{n}\left(\mathfrak{B}_{1} \times \cdots \times \mathfrak{B}_{n}\right)$.

A role of projective boundedness in harmonic analysis is described in section 4 . The terminology and results in this section will be used later in the paper.

In section 5 , it is shown by use of Grothendieck's (bilinear) inequality and factorization theorem that $\mathcal{F}_{2}=\mathcal{P} \mathcal{B F}_{2}$. In section 6 , it is established by use of a simple "tri-measure" due to C. Graham (e.g., [B5, p. 530], [GS2, proof of Corollary 4]) that $\mathcal{P B} \mathcal{F}_{3} \hookrightarrow \mathcal{F}_{3}$ is a proper imbedding. Existence of $\mu \in \mathcal{F}_{n}$ such that $\|\mu\|_{p b_{n}}=\infty$ can be deduced also from general results concerning multilinear Grothendieck-type inequalities ([V3], [B1]). In sections 7 and 8 , by the use of these results, projective boundedness is characterized within specific classes of $\mathcal{F}$-measures in frameworks of arbitrary dimension.

In a context of probability theory, just as classical "one-dimensional" measure theory is a natural setting for analysis of random variables, the multidimensional theory provides an effective setting for description and analysis of stochastic processes. This is illustrated in section 9 .

In section 10, I comment further on projective boundedness, and on its relation to $L^{2}$-factorizability, complete boundedness, and convolvability. Projective boundedness, a more stringent notion, is not implied by any of the last three. It implies convolvability, but whether it implies also $L^{2}$-factorizability or complete boundedness are open questions.

I have tried to make the paper accessible to probabilists as well as analysts. The requisites, which are stated in the next section, include the Grothendieck inequality and factorization theorem [G2]. For the convenience of readers, I have attached an appendix containing elementary proofs of these two fundamental results.

A major part of this paper was written during my stay at McGill University in the Fall of 1993; I thank S. Drury and I. Klemes for funding, through their NSERC grants, my enjoyable visit there.

\section{Preliminaries}

i. The algebras $V_{n}$ and $\widetilde{V}_{n}$ (cf. [V1], [V2], [GM, Chapter 11]). Throughout, the underlying scalar field will be the reals; modulo numerical constants, everything stated here holds equally well for complex scalars. $\mathcal{S}(\mathcal{X})$ will denote the space of simple scalar valued functions on a measurable space $(\mathcal{X}, \mathfrak{A})$. Let $\left(\mathcal{X}, \mathfrak{A}_{1}\right), \ldots,\left(\mathcal{X}_{n}, \mathfrak{A}_{n}\right)$ be measurable spaces, and let $\mathcal{S}\left(\mathcal{X}_{1}\right) \otimes \cdots \otimes \mathcal{S}\left(\mathcal{X}_{n}\right)$ denote the usual algebraic tensor product under the equivalence determined by pointwise equality on $\mathcal{X}_{1} \times \cdots \times \mathcal{X}_{n}$. The projective tensor norm of $\phi \in \mathcal{S}\left(\mathcal{X}_{1}\right) \otimes \cdots \otimes \mathcal{S}\left(\mathcal{X}_{n}\right)$ is

$$
\|\phi\|_{V_{n}}=\inf \left\{\sum_{j}\left\|\varphi_{1 j}\right\|_{\infty} \cdots\left\|\varphi_{n j}\right\|_{\infty} ; \phi=\sum_{j} \varphi_{1 j} \otimes \cdots \otimes \varphi_{n j}\right\} .
$$

The $V_{n}$-norm closure of $\mathcal{S}\left(\mathcal{X}_{1}\right) \otimes \cdots \otimes \mathcal{S}\left(\mathcal{X}_{n}\right)$ in $\mathcal{L}^{\infty}\left(\mathcal{X}_{1} \times \cdots \times \mathcal{X}_{n}\right)$ is the projective tensor product of $\mathcal{L}^{\infty}\left(\mathcal{X}_{1}\right), \ldots, \mathcal{L}^{\infty}\left(\mathcal{X}_{n}\right)$, denoted by $V_{n}\left(\mathcal{X}_{1} \times \cdots \times \mathcal{X}_{n}\right)$ or 
merely by $V_{n}$ when the underlying spaces $\mathcal{X}_{1}, \ldots, \mathcal{X}_{n}$ are understood from the context. Equipped with the $V_{n}$-norm and pointwise multiplication, $V_{n}$ is a Banach algebra. Traditionally, $V_{n}$ denotes the $n$-fold projective tensor product of spaces of continuous functions. Our point of view here abuses that custom only slightly. Indeed, $\mathcal{L}^{\infty}(\mathcal{X})$ can be canonically identified as a space of continuous functions on the maximal ideal space of $\mathcal{L}^{\infty}(\mathcal{X})$, in which case $V_{n}$, defined in the measurable setting above, has the traditional meaning.

The following are standard facts:

(i) If $f_{1} \in \mathcal{L}^{\infty}\left(\mathcal{X}_{1}\right), \ldots f_{n} \in \mathcal{L}^{\infty}\left(\mathcal{X}_{n}\right)$, then the elementary tensor $f_{1} \otimes \cdots \otimes f_{n}=\mathbf{f}$ is in $V_{n}$ and $\|\mathbf{f}\|_{V_{n}}=\|\mathbf{f}\|_{\infty}$.

(ii) $V_{n}=\left\{\phi \in \mathcal{L}^{\infty}\left(\mathcal{X}_{1} \times \cdots \times \mathcal{X}_{n}\right): \phi=\sum_{j=1}^{\infty} \mathbf{f}_{j}\right.$, the $\mathbf{f}_{j}$ 's are elementary tensors and $\left.\sum_{j=1}^{\infty}\left\|\mathbf{f}_{j}\right\|_{\infty}<\infty\right\}$.

Proposition 2.1. Suppose that $(\Omega, \nu)$ is a finite measure space. Let $g_{1} \in$ $\mathcal{L}^{\infty}\left(\mathcal{X}_{1}\right) \otimes L^{\infty}(\Omega), \ldots, g_{n} \in \mathcal{L}^{\infty}\left(\mathcal{X}_{n}\right) \otimes L^{\infty}(\Omega)$, and define $\phi \in \mathcal{L}^{\infty}\left(\mathcal{X}_{1} \times \cdots \times \mathcal{X}_{n}\right)$ by

$$
\phi\left(x_{1}, \ldots, x_{n}\right)=\int_{\Omega} g_{1}\left(x_{1}, \omega\right) \cdots g_{n}\left(x_{n}, \omega\right) \nu(d \omega) .
$$

Then $\phi \in V_{n}$, and

$$
\|\phi\|_{V_{n}} \leq\left\|g_{1}\right\|_{\infty} \cdots\left\|g_{n}\right\|_{\infty}\|\nu\|_{\mathcal{F}_{1}} .
$$

Let $\widetilde{V}_{n}\left(\mathcal{X}_{1} \times \cdots \times \mathcal{X}_{n}\right)=\widetilde{V}_{n}$ denote the algebra consisting of all pointwise limits of bounded sequences in $V_{n}\left(\mathcal{X}_{1} \times \cdots \times \mathcal{X}_{n}\right)$. Norm $\widetilde{V}_{n}$ by

$$
\|\phi\|_{\tilde{V}_{n}}=\inf \left\{\sup _{j}\left\|\varphi_{j}\right\|_{V_{n}}: \lim _{j \rightarrow \infty} \varphi_{j}(\mathbf{x})=\phi(\mathbf{x}), \mathbf{x} \in \mathcal{X}_{1} \times \cdots \times \mathcal{X}_{n}\right\} .
$$

The canonical imbedding $V_{n} \hookrightarrow \widetilde{V}_{n}$ is an isometry. It is a surjection if and only if at most one of the $\mathcal{X}_{j}$ 's is infinite (e.g., [V2]).

ii. $\mathcal{F}$-measures and duality. A $\mathcal{C}$-partition will mean a collection of pairwise disjoint elements of an algebra $\mathcal{C}$. If $\mathcal{C}_{1}, \ldots, \mathcal{C}_{n}$ are algebras of sets in $\mathcal{X}_{1}, \ldots, \mathcal{X}_{n}$, respectively, then a $\mathcal{C}_{1} \times \cdots \times \mathcal{C}_{n^{-}}$grid will mean an $n$-fold Cartesian product of finite $\mathcal{C}_{1}, \ldots, \mathcal{C}_{n}$-partitions. When $\mathcal{C}_{1}, \ldots, \mathcal{C}_{n}$ are understood from the context, we refer simply to partitions and grids. A Rademacher system indexed by a set $\tau$ is the collection of functions $\left\{r_{\alpha}\right\}_{\alpha \in \tau}$ defined on $\{-1,+1\}^{\tau}$, such that $r_{\alpha}(\omega)=\omega(\alpha)$ for $\alpha \in \tau$ and $\omega \in\{-1,+1\}^{\tau}$. If $\mu \in \mathcal{F}_{n}\left(\mathcal{C}_{1} \times \cdots \times \mathcal{C}_{n}\right)$, then the Fréchet variation of $\mu$ is defined by

$$
\|\mu\|_{\mathcal{F}_{n}}=\sup \left\{\left\|\sum_{\left(E_{1}, \ldots, E_{n}\right) \in \gamma} \mu\left(E_{1}, \ldots, E_{n}\right) r_{E_{1}} \otimes \cdots \otimes r_{E_{n}}\right\|_{\infty}: \operatorname{grid} \gamma\right\}
$$

$\left(r_{E_{1}}, \ldots, r_{E_{n}}\right.$ are indexed respectively by the partitions whose product is $\left.\gamma\right)$.

Theorem $2.2([\mathrm{~B} 7])$. Let $\mathcal{C}_{1}, \ldots, \mathcal{C}_{n}$ be algebras of sets in $\mathcal{X}_{1}, \ldots \mathcal{X}_{n}$, respectively. Denote $\mathfrak{A}_{1}=\sigma\left(\mathcal{C}_{1}\right), \ldots, \mathfrak{A}_{n}=\sigma\left(\mathcal{C}_{n}\right)$. Then $\mu \in \mathcal{F}_{n}\left(\mathcal{C}_{1} \times \cdots \times \mathcal{C}_{n}\right)$ determines an $\mathcal{F}_{n}$ measure on $\mathfrak{A}_{1} \times \cdots \times \mathfrak{A}_{n}$ if and only if $\|\mu\|_{F_{n}}<\infty$. Moreover, $\|\mu\|_{\mathcal{F}_{n}\left(\mathcal{C}_{1} \times \cdots \times \mathcal{C}_{n}\right)}=$ $\|\mu\|_{\mathcal{F}_{n}\left(\mathfrak{A}_{1} \times \cdots \times \mathfrak{A}_{n}\right)}$. 
Let $\left(\mathcal{X}_{1}, \mathfrak{A}_{1}\right), \ldots,\left(\mathcal{X}_{n}, \mathfrak{A}_{n}\right)$ be measurable spaces. Let $\phi \in \mathcal{S}\left(\mathcal{X}_{1}\right) \otimes \cdots \otimes \mathcal{S}\left(\mathcal{X}_{n}\right)$, and represent it as $\phi=\sum_{c \in \gamma} a_{c} \mathbf{1}_{c}$, where $\gamma$ is a grid and $\mathbf{1}_{c}$ denotes the indicator function of $c$. If $\mu$ is an $\mathcal{F}_{n}$-measure on $\mathfrak{A}_{1} \times \cdots \times \mathfrak{A}_{n}$, then define

$$
\int \phi d \mu:=\sum_{c \in \gamma} a_{c} \mu(c)
$$

and note

$$
\left|\int \phi d \mu\right| \leq\|\mu\|_{\mathcal{F}_{n}}\|\phi\|_{V_{n}} \text {. }
$$

The integral of $\phi \in V_{n}$ with respect to $\mu$ is obtained by taking a limit of the integrals defined by (2.6); the result obviously satisfies (2.7).

Proposition 2.3 ([Y1, Cor. 5.7], [B3, Lemma 4.9]). Let $\mu \in \mathcal{F}_{n}, f \in \mathcal{L}^{\infty}\left(\mathcal{X}_{1}\right)$, and define

$$
\mu_{f}\left(E_{2}, \ldots, E_{n}\right)=\int f(x) \mu\left(d x, E_{2}, \ldots, E_{n}\right), \quad\left(E_{2}, \ldots, E_{n}\right) \in \mathfrak{A}_{2} \times \cdots \times \mathfrak{A}_{n} .
$$

Then $\mu_{f} \in \mathcal{F}_{n-1}\left(\mathfrak{A}_{2} \times \cdots \times \mathfrak{A}_{n}\right)$ and

$$
\|\mu\|_{\mathcal{F}_{n-1}} \leq\|f\|_{\infty}\|\mu\|_{\mathcal{F}_{n}} .
$$

Proposition 2.3 implies that the integral of an elementary tensor can be computed iteratively:

$$
\int f_{1} \otimes \cdots \otimes f_{n} d \mu=\int f_{n}\left(x_{n}\right)\left(\ldots\left(\int f_{1}\left(x_{1}\right) \mu\left(d x_{1}, \ldots, d x_{n}\right)\right) \ldots\right) .
$$

The duality between the $V_{n}$ and $\mathcal{F}_{n}$ norms is an instance in a general scheme of tensor products ([G1], [G2], [DU, Chapter VIII]): if $f \in V_{n}$, then

$$
\|f\|_{V_{n}}=\sup \left\{\left|\int f d \mu\right|: \mu \in \mathcal{F}_{n},\|\mu\|_{\mathcal{F}_{n}} \leq 1\right\} \text {. }
$$

Induction (based on Proposition 2.3 and the Riesz representation theorem) implies

Theorem 2.4 ([Y1, Theorem 6.9], [B3, Theorem 4.12]). Let $\mathcal{X}_{1}, \ldots \mathcal{X}_{n}$ be locally compact Hausdorff spaces with respective Borel fields $\mathfrak{B}_{1}, \ldots, \mathfrak{B}_{n}$. Then every bounded $n$-linear functional on $\mathcal{C}_{0}\left(\mathcal{X}_{1}\right) \times \cdots \times \mathcal{C}_{0}\left(\mathcal{X}_{n}\right)$ uniquely determines an element in $\mathcal{F}_{n}\left(\mathfrak{B}_{1} \times \cdots \times \mathfrak{B}_{n}\right)$ such that the functional at $\left(f_{1}, \ldots, f_{n}\right) \in \mathcal{C}_{0}\left(\mathcal{X}_{1}\right) \times \cdots \times \mathcal{C}_{0}\left(\mathcal{X}_{n}\right)$ is the integral of $f_{1} \otimes \cdots \otimes f_{n}$ with respect to the corresponding $\mathcal{F}_{n}$-measure. In particular, if $V_{n}$ denotes the projective tensor product algebra $\mathcal{C}_{0}\left(\mathcal{X}_{1}\right) \hat{\otimes} \cdots \hat{\otimes} \mathcal{C}_{0}\left(\mathcal{X}_{n}\right)$, then $V_{n}^{*}=\mathcal{F}_{n}\left(\mathfrak{B}_{1} \times \cdots \times \mathfrak{B}_{n}\right)$.

iii. Grothendieck's theorem(s) [G2], [LP], [Pi] (see the Appendix to this paper)].

Theorem 2.5. There exists a universal constant $\mathcal{K}_{\mathcal{G}}>0$ with the following properties:

(i) (Inequality). Let $(\mathcal{X}, \mathfrak{A})$ and $(\mathcal{Y}, \mathfrak{B})$ be measurable spaces. If $\left(f_{j}\right)$ and $\left(g_{j}\right)$ are finite sequences in $\mathcal{L}^{\infty}(\mathcal{X})$ and $\mathcal{L}^{\infty}(\mathcal{Y})$, respectively, such that

$$
\sum_{j}\left|f_{j}(x)\right|^{2} \leq 1 \quad \text { and } \quad \sum_{j}\left|g_{j}(y)\right|^{2} \leq 1
$$


for $(x, y) \in \mathcal{X} \times \mathcal{Y}$, then

$$
\left\|\sum_{j} f_{j} \otimes g_{j}\right\|_{V_{2}} \leq \mathcal{K}_{\mathcal{G}} .
$$

(ii) (Factorization). If $\mathcal{X}$ and $\mathcal{Y}$ are locally compact Hausdorff spaces with respective Borel fields $\mathfrak{B}_{1}$ and $\mathfrak{B}_{2}$, and $\mu \in \mathcal{F}_{2}\left(\mathfrak{B}_{1} \times \mathfrak{B}_{2}\right)$, then there exist probability measure $\nu_{1}$ on $\mathfrak{B}_{1}$ and $\nu_{2}$ on $\mathfrak{B}_{2}$ such that for all $f \in \mathcal{C}_{0}(\mathcal{X})$ and $g \in \mathcal{C}_{0}(\mathcal{Y})$,

$$
\left|\int f \otimes g d \mu\right| \leq \mathcal{K}_{\mathcal{G}}\|\mu\|_{\mathcal{F}_{2}}\|f\|_{L^{2}\left(\nu_{1}\right)}\|g\|_{L^{2}\left(\nu_{2}\right)}
$$

3. $\mu$ IS PROJECTIVELY BOUNDED IF AND ONLY IF $\mu \times \nu \in \mathcal{F}_{n}$ FOR ALL $\nu \in \mathcal{F}_{n}$

Lemma 3.1. Let $\left(\mathcal{X}_{1}, \mathfrak{A}_{1}\right), \ldots,\left(\mathcal{X}_{n}, \mathfrak{A}_{n}\right),\left(\mathcal{Y}_{1}, \mathfrak{B}_{1}\right), \ldots,\left(\mathcal{Y}_{n}, \mathfrak{B}_{n}\right)$ be measurable spaces. Let $\mu \in \mathcal{F}_{n}\left(\mathfrak{A}_{1} \times \cdots \times \mathfrak{A}_{n}\right)$ and $\nu \in \mathcal{F}_{n}\left(\mathfrak{B}_{1} \times \cdots \times \mathfrak{B}_{n}\right)$. Extend the domain of $\mu \times \nu($ see $(1.1))$ to include $a\left(\mathfrak{A}_{1} \times \mathfrak{B}_{1}\right) \times \cdots \times a\left(\mathfrak{A}_{n} \times \mathfrak{B}_{n}\right)(a(\mathfrak{A} \times \mathfrak{B})$ denotes the algebra generated by $\mathfrak{A} \times \mathfrak{B})$. Then $\mu \times \nu$ determines an $\mathcal{F}_{n}$-measure on $\sigma\left(\mathfrak{A}_{1} \times \mathfrak{B}_{1}\right) \times \cdots \times \sigma\left(\mathfrak{A}_{n} \times \mathfrak{B}_{n}\right)$ if and only if

$$
:=\sup \left\{\begin{array}{c}
\|\mu \times \nu\|_{\mathcal{F}_{n}\left(a\left(\mathfrak{A}_{1} \times \mathfrak{B}_{1}\right) \times \cdots \times a\left(\mathfrak{A}_{n} \times \mathfrak{B}_{n}\right)\right)}:= \\
\sum_{\substack{\left(A_{1}, \ldots, A_{n}\right) \in \alpha \\
\left(B_{1}, \ldots, B_{n}\right) \in \beta}} \mu \times \nu\left(\left(A_{1}, B_{1}\right), \ldots,\left(A_{n}, B_{n}\right)\right) r_{\left(A_{1}, B_{1}\right)} \otimes \cdots \otimes r_{\left(A_{n}, B_{n}\right)} \|_{\infty}:
\end{array}\right.
$$

$$
\left.\operatorname{grid} \alpha \text { of } \mathcal{X}_{1} \times \cdots \times \mathcal{X}_{n}, \operatorname{grid} \beta \text { of } \mathcal{Y}_{1} \times \mathcal{Y}_{n}\right\}
$$

is finite. In particular, if $\mu \times \nu \in \mathcal{F}_{n}\left(\sigma\left(\mathfrak{A}_{1} \times \mathfrak{B}_{1}\right) \times \cdots \times \sigma\left(\mathfrak{A}_{n} \times \mathfrak{B}_{n}\right)\right)$, then (3.1) equals $\|\mu \times \nu\|_{\mathcal{F}_{n}}$ (if the extension of $\mu \times \nu$ exists, then it is denoted also by $\mu \times \nu$ ).

Proof. The only if direction follows from the only if direction of Theorem 2.2.

To establish the converse, observe that Theorem 1.3 in the (classical) case $n=1$ implies that $\mu \times \nu \in \mathcal{F}_{n}\left(a\left(\mathfrak{A}_{1} \times \mathfrak{B}_{1}\right) \times \cdots \times a\left(\mathfrak{A}_{n} \times \mathfrak{B}_{n}\right)\right)$, and then apply the if direction of Theorem 2.2.

Lemma 3.2. Let $\left(\mathcal{X}_{1}, \mathfrak{A}_{1}\right), \ldots,\left(\mathcal{X}_{n}, \mathfrak{A}_{n}\right)$ be measurable spaces. If

$$
\mu \in \mathcal{F}_{n}\left(\mathfrak{A}_{1} \times \cdots \times \mathfrak{A}_{n}\right)
$$

and $\phi_{\mu}$ is defined by (1.2), then

$$
\begin{array}{r}
\|\mu\|_{p b_{n}}=\sup \left\{\left\|\phi_{\mu}\right\|_{V_{n}\left(F_{1} \times \cdots \times F_{n}\right)}: F_{i} \subset \text { unit ball of } \mathcal{S}\left(\mathcal{X}_{i}\right),\right. \\
\left.\qquad\left|F_{i}\right|<\infty, i=1, \ldots, n\right\} .
\end{array}
$$

Proof. That $\|\mu\|_{p b_{n}}$ majorizes the right side of (3.2) is obvious.

For each $j=1, \ldots, n$, let $F_{j} \subset \mathbb{N}$ be finite and let $\left\{f_{j k}: k \in F_{j}\right\}$ be an arbitrary subset of the unit ball of $\mathcal{L}^{\infty}\left(\mathcal{X}_{j}\right)$. For each $k \in F_{j}$, let $\left\{g_{m j k}: m \in \mathbb{N}\right\}$ be a 
sequence of simple functions in the unit ball of $\mathcal{L}^{\infty}\left(\mathcal{X}_{j}\right)$ uniformly converging to $f_{j k}$. Define

$$
\begin{aligned}
\phi_{\mu}\left(k_{1}, \ldots, k_{n}\right) & =\int f_{j k_{1}} \otimes \cdots \otimes f_{j k_{n}} d \mu, \\
\phi_{\mu}^{(m)}\left(k_{1}, \ldots, k_{n}\right) & =\int g_{m j k_{1}} \otimes \cdots \otimes g_{m j k_{n}} d \mu,
\end{aligned}
$$

for $\left(k_{1}, \ldots, k_{n}\right) \in F_{1} \times \cdots \times F_{n}$. By $(2.7),\left\{\phi_{\mu}^{(m)}: m \in \mathbb{N}\right\}$ converges pointwise to $\phi_{\mu}$ on $F_{1} \times \cdots \times F_{n}$. This implies

$$
\text { right side of }(3.2) \geq\left\|\phi_{\mu}\right\|_{\tilde{V}_{n}}
$$

(see (2.4)). Since the $F_{j}$ 's are finite, $\phi_{\mu} \in V_{n}\left(F_{1} \times \cdots \times F_{n}\right)$ and $\left\|\phi_{\mu}\right\|_{\widetilde{V}_{n}}=\left\|\phi_{\mu}\right\|_{V_{n}}$. This, by (3.4), implies that the right side of (3.2) majorizes $\|\mu\|_{p b_{n}}$.

Proof of Theorem 1.3. If $\mu \in \mathcal{F}_{n}\left(\mathfrak{A}_{1} \times \cdots \times \mathfrak{A}_{n}\right)$ and $f_{1} \in \mathcal{L}^{\infty}\left(\mathcal{X}_{1} \times \mathcal{Y}_{1}\right), \ldots, f_{n} \in$ $\mathcal{L}^{\infty}\left(\mathcal{X}_{n} \times \mathcal{Y}_{n}\right)$, then denote

$$
\phi_{f_{1} \cdots f_{n} ; \mu}\left(y_{1}, \ldots, y_{n}\right)=\int f_{1}\left(x_{1}, y_{1}\right) \cdots f_{n}\left(x_{n}, y_{n}\right) \mu\left(d x_{1}, \ldots, d x_{n}\right),
$$

$\left(y_{1}, \ldots, y_{n}\right) \in \mathcal{Y}_{1} \times \cdots \times \mathcal{Y}_{n}$. Lemma 3.2 implies

$$
\|\mu\|_{p b_{n}}=\sup \left\{\left\|\phi_{f_{1} \cdots f_{n} ; \mu}\right\|_{V_{n}}: f_{i} \in \mathcal{S}\left(\mathcal{X}_{i}\right) \otimes \mathcal{S}\left(\mathcal{Y}_{i}\right),\left\|f_{i}\right\|_{\infty} \leq 1, i=1, \ldots, n\right\} .
$$

Suppose $\|\mu\|_{p b_{n}}<\infty$, and let $\nu \in \mathcal{F}_{n}\left(\mathfrak{B}_{1} \times \cdots \times \mathfrak{B}_{n}\right)$. Let $\alpha_{1}, \ldots, \alpha_{n}$, and $\beta_{1}, \ldots, \beta_{n}$ be finite partitions of $\mathcal{X}_{1}, \ldots, \mathcal{X}_{n}$ and $\mathcal{Y}_{1}, \ldots, \mathcal{Y}_{n}$, respectively. Let $\boldsymbol{\alpha}=$ $\alpha_{1} \times \cdots \times \alpha_{n}$ and $\boldsymbol{\beta}=\beta_{1} \times \cdots \times \beta_{n}$ be the resulting grids. Fix $\omega_{i} \in\{-1,+1\}^{\alpha_{i} \times \beta_{i}}$ and define

$$
f_{i}=\sum_{(A, B) \in \alpha_{i} \times \beta_{i}} r_{(A, B)}\left(\omega_{i}\right) \mathbf{1}_{A \times B}
$$

Then

$$
\begin{gathered}
\left|\sum_{\substack{\left(A_{1}, \ldots, A_{n}\right) \in \boldsymbol{\alpha} \\
\left(B_{1}, \ldots, B_{n}\right) \in \boldsymbol{\beta}}} \mu \times \nu\left(\left(A_{1}, B_{1}\right), \ldots,\left(A_{n}, B_{n}\right)\right) r_{\left(A_{1}, B_{1}\right)}\left(\omega_{1}\right) \cdots r_{\left(A_{n}, B_{n}\right)}\left(\omega_{n}\right)\right| \\
=\left|\int \phi_{f_{1} \cdots f_{n} ; \mu} d \nu\right| \leq\|\mu\|_{p b_{n}}\|\nu\|_{\mathcal{F}_{n}} .
\end{gathered}
$$

By Lemma 3.1, $\mu \times \nu$ determines an $\mathcal{F}_{n}$-measure on $\sigma\left(\mathfrak{A}_{1} \times \mathfrak{B}_{1}\right) \times \cdots \times \sigma\left(\mathfrak{A}_{n} \times \mathfrak{B}_{n}\right)$.

Conversely, suppose that $\mu \times \nu \in \mathcal{F}_{n}\left(\sigma\left(\mathfrak{A}_{1} \times \mathfrak{B}_{1}\right) \times \cdots \times \sigma\left(\mathfrak{A}_{n} \times \mathfrak{B}_{n}\right)\right)$ for every $\nu \in \mathcal{F}_{n}\left(\mathfrak{B}_{1} \times \cdots \times \mathfrak{B}_{n}\right)$. Then there exists $\mathcal{K}>0$ such that

$$
\|\mu \times \nu\|_{\mathcal{F}_{n}} \leq \mathcal{K}\|\nu\|_{\mathcal{F}_{n}} \quad \text { for all } \nu \in \mathcal{F}_{n}\left(\mathfrak{B}_{1} \times \cdots \times \mathfrak{B}_{n}\right) .
$$

For each $i=1, \ldots, n$, let $f_{i}$ be a simple function on $\mathcal{X}_{i} \times \mathcal{Y}_{i}$ of the form

$$
f_{i}=\sum_{(A, B) \in \alpha_{i} \times \beta_{i}} a_{(A, B)} \mathbf{1}_{A \times B},
$$


where $\alpha_{i}$ and $\beta_{i}$ are partitions of $\mathcal{X}_{i}$ and $\mathcal{Y}_{i}$, respectively. Note that

$$
\begin{aligned}
\int \phi_{f_{1} \cdots f_{n} ; \mu} d \nu \\
=\sum_{\substack{\left(A_{1}, \ldots, A_{n}\right) \in \boldsymbol{\alpha} \\
\left(B_{1}, \ldots, B_{n}\right) \in \boldsymbol{\beta}}} \mu \times \nu\left(\left(A_{1}, B_{1}\right), \ldots,\left(A_{n}, B_{n}\right)\right) a_{\left(A_{1}, B_{1}\right)} \cdots a_{\left(A_{n}, B_{n}\right)} .
\end{aligned}
$$

Then, by (3.9) and duality (see (2.11)), we obtain

$$
\left\|\phi_{f_{1} \cdots f_{n} ; \mu}\right\|_{V_{n}} \leq \mathcal{K}\left\|f_{1}\right\|_{\infty} \cdots\left\|f_{n}\right\|_{\infty}
$$

which, by Lemma 3.2 , implies $\|\mu\|_{p b_{n}} \leq \mathcal{K}$.

Corollary 3.3. If $\mu \in \mathcal{P} \mathcal{B F}_{n}\left(\mathfrak{A}_{1} \times \cdots \times \mathfrak{A}_{n}\right)$ and $\nu \in \mathcal{F}_{n}\left(\mathfrak{B}_{1} \times \cdots \times \mathfrak{B}_{n}\right)$, then

$$
\|\mu \times \nu\|_{\mathcal{F}_{n}} \leq\|\mu\|_{p b_{n}}\|\nu\|_{\mathcal{F}_{n}}
$$

\section{Harmonic analysis of $\mathcal{F}$-MEasures}

Let $\mathcal{X}_{1}, \ldots, \mathcal{X}_{n}$ be locally compact Abelian groups with Borel fields $\mathfrak{B}_{1}, \ldots, \mathfrak{B}_{n}$, and let $\widehat{\mathcal{X}}_{1}, \ldots, \widehat{\mathcal{X}}_{n}$ be the respective dual groups with Borel fields $\widehat{\mathfrak{B}}_{1}, \ldots, \widehat{\mathfrak{B}}_{n}$. If $\mu$ is a bounded multilinear functional on $\mathcal{C}_{0}\left(\mathcal{X}_{1}\right) \times \cdots \times \mathcal{C}_{0}\left(\mathcal{X}_{n}\right)$, then $\mu$ is identified as an $\mathcal{F}_{n}$-measure on $\mathfrak{B}_{1} \times \cdots \times \mathfrak{B}_{n}$ (Theorem 2.4), and its transform $\hat{\mu}$ is then defined by

$$
\hat{\mu}\left(\gamma_{1}, \ldots, \gamma_{n}\right)=\int \gamma_{1} \otimes \cdots \otimes \gamma_{n} d \mu, \quad\left(\gamma_{1}, \ldots, \gamma_{n}\right) \in \widehat{\mathcal{X}}_{1} \times \cdots \times \widehat{\mathcal{X}}_{n} .
$$

It is evident that $\hat{\mu}$ is continuous separately in each coordinate and is bounded by $\|\mu\|_{\mathcal{F}_{n}}$.

Proposition 4.1. If $\mu \in \mathcal{P} \mathcal{B} \mathcal{F}_{n}\left(\mathfrak{B}_{1} \times \cdots \times \mathfrak{B}_{n}\right)$, then $\hat{\mu} \in \widetilde{V}_{n}\left(\widehat{\mathcal{X}}_{1} \times \cdots \times \widehat{\mathcal{X}}_{n}\right)$. In particular,

$$
\|\hat{\mu}\|_{\widetilde{V}_{n}} \leq\|\mu\|_{p b_{n}} .
$$

Proof. It suffices to establish that if $\mathcal{K}_{1} \subset \widehat{\mathcal{X}}_{1}, \ldots, \mathcal{K}_{n} \subset \widehat{\mathcal{X}}_{n}$ are compact, then

$$
\|\hat{\mu}\|_{\widetilde{V}_{n}\left(\mathcal{K}_{1} \times \cdots \times \mathcal{K}_{n}\right)} \leq\|\mu\|_{p b_{n}}
$$

$\hat{\mu}$ is uniformly approximable on $\mathcal{K}_{1} \times \cdots \times \mathcal{K}_{n}$ by simple functions

$$
\phi=\sum_{\gamma \in F_{1}, \ldots, \gamma_{n} \in F_{n}} \hat{\mu}\left(\gamma_{1}, \ldots, \gamma_{n}\right) \mathbf{1}_{\tau_{1}\left(\gamma_{1}\right)} \otimes \cdots \otimes \mathbf{1}_{\tau_{n}\left(\gamma_{n}\right)},
$$

where $F_{1} \subset \mathcal{K}_{1}, \ldots, F_{n} \subset \mathcal{K}_{n}$ are finite sets, and $\tau_{1}, \ldots, \tau_{n}$ are $\widehat{\mathfrak{B}}_{1}, \ldots, \widehat{\mathfrak{B}}_{n}$-partitions indexed respectively by $F_{1}, \ldots, F_{n}$. Note that $\phi$, defined by (4.4) above, can be identified with $\phi_{\mu}$ which is defined by (1.2). Therefore, $\|\phi\|_{V_{n}\left(\mathcal{K}_{1} \times \cdots \times \mathcal{K}_{n}\right)} \leq\|\mu\|_{p b_{n}}$, which implies (4.3).

If $\mu \in \mathcal{P B} \mathcal{F}_{n}\left(\mathfrak{B}_{1} \times \cdots \times \mathfrak{B}_{n}\right)$ and $\nu \in \mathcal{F}_{n}\left(\mathfrak{B}_{1} \times \cdots \times \mathfrak{B}_{n}\right)$, then the convolution $\mu \star \nu$ is obtained by mimicking a standard definition (e.g., [R, 1.3.1]):

$$
\begin{array}{r}
\mu \star \nu\left(E_{1}, \ldots, E_{n}\right)=\int \mathbf{1}_{E_{1}}\left(x_{1}+y_{1}\right) \cdots \mathbf{1}_{E_{n}}\left(x_{n}+y_{n}\right) \mu \times \nu\left(d\left(x_{1}, y_{1}\right), \ldots, d\left(x_{n}, y_{n}\right)\right), \\
E_{1} \in \mathfrak{B}_{1}, \ldots, E_{n} \in \mathfrak{B}_{n} .
\end{array}
$$


Proposition 4.2. If $\mu \in \mathcal{P B F}_{n}\left(\mathfrak{B}_{1} \times \cdots \times \mathfrak{B}_{n}\right)$ and $\nu \in \mathcal{F}_{n}\left(\mathfrak{B}_{1} \times \cdots \times \mathfrak{B}_{n}\right)$, then $\mu \star \nu \in \mathcal{F}_{n}\left(\mathfrak{B}_{1} \times \cdots \times \mathfrak{B}_{n}\right)$. Moreover,

$$
\|\mu \star \nu\|_{\mathcal{F}_{n}} \leq\|\mu\|_{p b_{n}}\|\nu\|_{\mathcal{F}_{n}}
$$

and

$$
(\mu \star \nu)^{\wedge}=\hat{\mu} \hat{\nu} .
$$

Proof. The first assertion follows via (2.10) from the usual dominated convergence theorem. (4.6) follows from (3.13). To establish (4.7), note that if $f_{i} \in \mathcal{L}^{\infty}\left(\mathcal{X}_{i}\right)$ and $g_{i} \in \mathcal{L}^{\infty}\left(\mathcal{X}_{i}\right)(i=1, \ldots, n)$, then

$$
\begin{aligned}
\int f_{1} & \otimes g_{1} \otimes \cdots \otimes f_{n} \otimes g_{n} d(\mu \times \nu) \\
= & \left(\int f_{1} \otimes \cdots \otimes f_{n} d \mu\right)\left(\int g_{1} \otimes \cdots \otimes g_{n} d \nu\right),
\end{aligned}
$$

and

$$
\begin{aligned}
& \int f_{1} \otimes \cdots \otimes f_{n} d(\mu \star \nu) \\
& \quad=\int f_{1}\left(x_{1}+y_{1}\right) \cdots f_{n}\left(x_{n}+y_{n}\right) \mu \times \nu\left(d\left(x_{1}, y_{1}\right), \ldots, d\left(x_{n}, y_{n}\right)\right) .
\end{aligned}
$$

\section{Every $\mu \in \mathcal{F}_{2}$ IS PROJECTIVELY Bounded}

Theorem 5.1. If $(\mathcal{X}, \mathfrak{A})$ and $(\mathcal{Y}, \mathfrak{B})$ are measurable spaces, then $\mathcal{P B F}_{2}(\mathfrak{A} \times \mathfrak{B})=$ $\mathcal{F}_{2}(\mathfrak{A} \times \mathfrak{B})$. Specifically, if $\mu \in \mathcal{F}_{2}(\mathfrak{A} \times \mathfrak{B})$, then $\|\mu\|_{p b_{2}} \leq\left(\mathcal{K}_{\mathcal{G}}\right)^{2}\|\mu\|_{\mathcal{F}_{2}}$, where $\mathcal{K}_{\mathcal{G}}>0$ is the Grothendieck constant (see Theorem 2.5).

Proof. We need to show that if $F$ and $G$ are finite sets in the respective unit balls of $\mathcal{S}(\mathcal{X})$ and $\mathcal{S}(\mathcal{Y})$, and

$$
\phi(f, g)=\int f \otimes g d \mu, \quad(f, g) \in F \times G
$$

then

$$
\|\phi\|_{V_{2}(F \times G)} \leq\left(\mathcal{K}_{\mathcal{G}}\right)^{2}\|\mu\|_{\mathcal{F}_{2}}
$$

(see Lemma 3.2). There exist finite partitions $\mathcal{C}$ and $\mathcal{D}$ of $\mathcal{X}$ and $\mathcal{Y}$, respectively, such that for every $f \in F$ and $g \in G$,

$$
f=\sum_{c \in \mathcal{C}} f(c) \mathbf{1}_{c}, \quad g=\sum_{d \in \mathcal{D}} g(d) \mathbf{1}_{d}
$$

( $f(c)$ and $g(d)$ are the constant values assumed by $f$ and $g$ on $c$ and $d$ in the respective partitions). Then (5.1) can be rewritten as

$$
\phi(f, g)=\sum_{(c, d) \in \mathcal{C} \times \mathcal{D}} \mu(c, d) f(c) g(d), \quad f \in F, g \in G .
$$

Let $\left.\mu\right|_{\mathcal{C} \times \mathcal{D}}$ denote the restriction of $\mu$ to $\sigma(\mathcal{C}) \times \sigma(\mathcal{D})$. Then

$$
\left\|\left.\mu\right|_{\mathcal{C} \times \mathcal{D}}\right\|_{\mathcal{F}_{2}} \leq\|\mu\|_{\mathcal{F}_{2}} \text {. }
$$


In Theorem 2.5(ii), let $\mathcal{X}=\mathcal{C}$ and $\mathcal{Y}=\mathcal{D}$, and let the action of $\beta$ be integration with respect to $\left.\mu\right|_{\mathcal{C} \times \mathcal{D}}$. Then conclude that there exist probability measures $\nu_{1}$ on $\mathcal{C}$ and $\nu_{2}$ on $\mathcal{D}$ such that

$$
\sup \left\{\left|\sum_{(c, d) \in \mathcal{C} \times \mathcal{D}} \mu\right|_{\mathcal{C} \times \mathcal{D}} f(c) g(d) \mid:\|f\|_{L^{2}\left(\nu_{1}\right)} \leq 1,\|g\|_{L^{2}\left(\nu_{1}\right)} \leq 1\right\} \leq \mathcal{K}_{\mathcal{G}}\|\mu\|_{\mathcal{F}_{2}} .
$$

Theorem 2.5(i) is equivalent to the following:

Let $\mathcal{H}_{1}$ and $\mathcal{H}_{2}$ be Hilbert spaces, and let $\eta$ be a bilinear functional on $\mathcal{H}_{1} \times \mathcal{H}_{2}$. If $F$ and $G$ are finite subsets of the respective unit balls of $\mathcal{H}_{1}$ and $\mathcal{H}_{2}$, then

$$
\|\eta\|_{V_{2}(F \times G)} \leq \mathcal{K}_{\mathcal{G}} \sup \left\{|\eta(\mathbf{x}, \mathbf{y})|:\|\mathbf{x}\|_{\mathcal{H}_{1}} \leq 1,\|\mathbf{y}\|_{\mathcal{H}_{2}} \leq 1\right\} .
$$

(To see this, without loss of generality take $\mathcal{H}_{1}$ and $\mathcal{H}_{2}$ to be $l^{2}$ and let $\eta$ be the usual inner product in $l^{2}$; then, in the statement of Theorem 2.5(i), take $\mathcal{X}=F, \mathcal{Y}=G$, and $f_{j}(\mathbf{x})=\mathbf{x}(j), g_{j}(\mathbf{y})=\mathbf{y}(j)$.) In (5.5), let $\mathcal{H}_{1}=L^{2}\left(\mathcal{C}, \nu_{1}\right), \mathcal{H}_{2}=L^{2}\left(\mathcal{D}, \nu_{2}\right)$, $\eta=\phi$, and thus obtain (5.2) from (5.4).

Remark. If $(\mathcal{X}, \mathfrak{A})$ and $(\mathcal{Y}, \mathfrak{B})$ are locally compact abelian groups, then Theorem 5.1 and Proposition 4.2 directly imply that $\mathcal{F}_{2}(\mathfrak{A} \times \mathfrak{B})$ has a convolution structure which extends the usual convolution structure in $\mathcal{F}_{1}(\sigma(\mathfrak{A} \times \mathfrak{B}))$. This was proved first in [GS1], also by an application of Grothendieck's theorem(s) (see also [GiIS]).

\section{There exist Projectively unbounded $\mathcal{F}_{3}$-Measures}

Theorem 6.1. If $(\mathcal{X}, \mathfrak{A}),(\mathcal{Y}, \mathfrak{B})$, and $(\mathcal{Z}, \mathfrak{C})$ are measurable spaces with infinite underlying $\sigma$-algebras, then there exists $\mu \in \mathcal{F}_{3}(\mathfrak{A} \times \mathfrak{B} \times \mathfrak{C})$ such that $\|\mu\|_{p b_{3}}=\infty$.

In order to prove Theorem 6.1, we establish first a finite version of it. Fix a positive integer $m$. Let $\mathbb{Z}_{m}$ denote the additive group of integers $\bmod m$, endowed with the uniform probability measure (Haar measure). Then $\widehat{\mathbb{Z}}_{m}$ (the character group of $\left.\mathbb{Z}_{m}\right)$ is canonically identified with $\mathbb{Z}_{m}$. Let $\psi_{m}$ be the function on $\mathbb{Z}_{m} \times \widehat{\mathbb{Z}}_{m}$ representing the dual action between $\widehat{\mathbb{Z}}_{m}$ and $\mathbb{Z}_{m}$, i.e.,

$$
\psi_{m}(k, l)=e^{2 \pi i k l / m}, \quad(k, l) \in \mathbb{Z}_{m} \times \widehat{\mathbb{Z}}_{m} .
$$

It is a standard fact that for $\alpha \in l^{2}\left(\mathbb{Z}_{m}\right)$ and $\beta \in l^{2}\left(\widehat{\mathbb{Z}}_{m}\right)$,

$$
\left|\sum_{(k, l) \in \mathbb{Z}_{m} \times \widehat{\mathbb{Z}}_{m}} \sqrt{\frac{1}{m}} \psi_{m}(k, l) \alpha(k) \beta(l)\right| \leq\|\alpha\|_{2}\|\beta\|_{2},
$$

which implies

$$
\left\|\left(1 / m^{3 / 2}\right) \psi_{m}\right\|_{\mathcal{F}_{2}} \leq 1
$$

(e.g., cf. [L, p. 172]). This, in turn, implies

$$
\sqrt{m} \leq\left\|\psi_{m}\right\|_{V_{2}}
$$

Define an $\mathcal{F}_{3}$-measure $\mu$ on $\mathbb{Z}_{m} \times \mathbb{Z}_{m} \times \widehat{\mathbb{Z}}_{m}$ by

$$
\mu(A, B, C)=\sum_{(k, l) \in \widehat{\mathbb{Z}}_{m} \times \mathbb{Z}_{m}} \psi_{m}(k, l) \hat{\mathbf{1}}_{A}(k) \hat{\mathbf{1}}_{B}(k) \hat{\mathbf{1}}_{C}(l),
$$

where $A \subset \mathbb{Z}_{m}, B \subset \mathbb{Z}_{m}, C \subset \widehat{\mathbb{Z}}_{m}$. 
Lemma 6.2. $\|\mu\|_{\mathcal{F}_{3}} \leq 1$ and $\|\mu\|_{p b_{3}} \geq \sqrt{m}$.

Proof. If $f$ and $g$ are in $l^{\infty}\left(\mathbb{Z}_{m}\right)$ and $h$ is in $l^{\infty}\left(\widehat{\mathbb{Z}}_{m}\right)$, then

$$
\left|\int f \otimes g \otimes h d \mu\right|=\sum_{(k, l) \in \widehat{\mathbb{Z}}_{m} \times \mathbb{Z}_{m}} \psi_{m}(k, l) \hat{f}(k) \hat{g}(k) \hat{h}(l)=\left|\sum_{k \in \widehat{\mathbb{Z}}_{m}} \hat{f}(k) \hat{g}(k) h(k)\right|
$$

(by the inversion of the Fourier transform)

$$
\leq\|\hat{f}\|_{2}\|\hat{g}\|_{2}\|h\|_{\infty} \leq\|f\|_{\infty}\|g\|_{\infty}\|h\|_{\infty}
$$

which implies $\|\mu\|_{\mathcal{F}_{3}} \leq 1$.

To obtain the second inequality, note that the transform $\hat{\mu}$ (cf. (4.1)) is given by

$$
\hat{\mu}\left(k_{1}, k_{2}, l\right)= \begin{cases}\psi(k, l) & \text { if } k_{1} \in \mathbb{Z}_{m}, k_{2} \in \mathbb{Z}_{m}, k_{1}=k_{2}=k, l \in \widehat{\mathbb{Z}}_{m}, \\ 0 & \text { otherwise. }\end{cases}
$$

In the definition of projective boundedness, let $F_{1}=F_{2}=\widehat{\mathbb{Z}}_{m}$ (characters on $\mathbb{Z}_{m}$ ) and let $F_{3}=\mathbb{Z}_{m}$ (characters on $\left.\widehat{\mathbb{Z}}_{m}\right)$, and then deduce $\|\nu\|_{p b_{3}} \geq\|\hat{\mu}\|_{V_{3}\left(\widehat{\mathbb{Z}}_{m} \times \widehat{\mathbb{Z}}_{m} \times \mathbb{Z}_{m}\right)}$ (cf. Proposition 4.1). Therefore, by (6.7) and (6.4),

$$
\|\mu\|_{p b_{3}} \geq\|\hat{\mu}\|_{V_{3}\left(\widehat{\mathbb{Z}}_{m} \times \widehat{\mathbb{Z}}_{m} \times \mathbb{Z}_{m}\right)} \geq\left\|\psi_{m}\right\|_{V_{2}\left(\widehat{\mathbb{Z}}_{m} \times \mathbb{Z}_{m}\right)} \geq \sqrt{m} .
$$

Proof of Theorem 6.1. For $j \in \mathbb{N}$, let $\mathcal{A}_{j} \subset \mathfrak{A}, \mathcal{B}_{j} \subset \mathfrak{B}$, and $\mathcal{C}_{j} \subset \mathfrak{C}$ be partitions such that $\left|\mathcal{A}_{j}\right|=\left|\mathcal{B}_{j}\right|=\left|\mathcal{C}_{j}\right|=2^{j}$, and such that the $\mathcal{A}_{j}$ 's, $\mathcal{B}_{j}$ 's, and $\mathcal{C}_{j}$ 's are pairwise disjoint. For each $j \in \mathbb{N}$, and $a \in \mathcal{A}_{j}, b \in \mathcal{B}_{j}$, and $c \in \mathcal{C}_{j}$, choose points $x_{j}(a) \in a, y_{j}(b) \in b$, and $z_{j}(c) \in c$. Identify $\mathcal{A}_{j}$ and $\mathcal{B}_{j}$ with $\mathbb{Z}_{2^{j}}$, and identify $\mathcal{C}_{j}$ with $\widehat{\mathbb{Z}}_{2^{j}}$. Following this identification, apply Lemma 6.2 with $m=2^{j}$, thus obtaining $\mu_{j} \in \mathcal{F}_{3}\left(\sigma\left(\mathcal{A}_{j}\right) \times \sigma\left(\mathcal{B}_{j}\right) \times \sigma\left(\mathcal{C}_{j}\right)\right)$ such that

$$
\left\|\mu_{j}\right\|_{\mathcal{F}_{3}} \leq 1 \text { and }\left\|\mu_{j}\right\|_{p b_{3}} \geq 2^{j / 2} .
$$

Extend the $\mu_{j}$ 's to $\mathfrak{A} \times \mathfrak{B} \times \mathfrak{C}$ by defining, for $(A, B, C) \in \mathfrak{A} \times \mathfrak{B} \times \mathfrak{C}$,

$$
\mu_{j}(A, B, C)= \begin{cases}\mu_{j}(a, b, c), & \text { if } x_{j}(a) \in A, y_{j}(b) \in B, z_{j}(c) \in C, \\ 0, & \text { otherwise. }\end{cases}
$$

Note that the $\mu_{j}$ 's still satisfy (6.9). Let $\mu=\sum_{j} \mu_{j} / j^{2}$. Then $\mu \in \mathcal{F}_{3}(\mathfrak{A} \times \mathfrak{B} \times \mathfrak{C})$, and

$$
\|\mu\|_{p b_{3}} \geq\left\|\mu_{j}\right\|_{p b_{3}} / j^{2} \geq 2^{j / 2} / j^{2} \quad \text { for all } j \in \mathbb{N} \text {. }
$$

\section{A ChaRACTERIZATION OF PROJECTIVE BOUNDEDNESS}

In this and the next section, $\mathcal{F}$-measures will be viewed in a framework of harmonic analysis. To set the stage, we first recall the multilinear Grothendieck-type inequalities derived in [B1].

Definition 7.1 ([B1]). An $n$-linear form $\mathcal{A}$ on a Hilbert space $\mathcal{H}$ is projectively bounded if

$$
\begin{aligned}
\|\mathcal{A}\|_{p b_{n}}:=\sup \left\{\|\mathcal{A}\|_{V_{n}\left(F_{1} \times \cdots \times F_{n}\right)}:\right. & F_{i} \subset \text { unit ball of } \mathcal{H}, \\
& \left.\left|F_{i}\right|<\infty, i=1, \ldots, n\right\}<\infty .
\end{aligned}
$$


Fix $m \in \mathbb{N}$. Let $U=\left\{S_{\alpha}: \alpha=1, \ldots, n\right\}$ be a collection of ordered subsets of $[m](:=\{1, \ldots, m\})$ such that $U$ covers $[m]$ and every $j \in[m]$ appears in at least two elements of $U$. Denote the cardinality of $S_{\alpha}$ by $k_{\alpha}$, and define $\pi_{\alpha}: \mathbb{N}^{m} \rightarrow \mathbb{N}^{k_{\alpha}}$ by

$$
\pi_{\alpha}\left(l_{1}, \ldots, l_{m}\right)=\left(l_{i}: i \in S_{\alpha}\right), \quad \alpha \in[n] .
$$

Let $\mathcal{H}$ be a separable Hilbert space, and let $\mathcal{B}$ be an orthonormal basis of $\mathcal{H}$. For each $\alpha \in[n]$, fix a $k_{\alpha}$-dimensional enumeration of $\mathcal{B}$, i.e.,

$$
\mathcal{B}_{\alpha}(=\mathcal{B})=\left\{e_{\mathbf{j}}: \mathbf{j} \in \mathbb{N}^{k_{\alpha}}\right\} .
$$

For each $\alpha$, let $\mathcal{H}_{\alpha}$ designate the Hilbert space $\mathcal{H}$ spanned by $\mathcal{B}_{\alpha}$, i.e.,

$$
x \sim \sum_{\mathbf{j} \in \mathbb{N}^{k_{\alpha}}} x(\mathbf{j}) e_{\mathbf{j}}, \quad x \in \mathcal{H} .
$$

For $\varphi \in l^{\infty}\left(\mathbb{N}^{m}\right)$, define

$$
\mathcal{A}_{\varphi}\left(x_{1}, \ldots, x_{n}\right)=\sum_{\boldsymbol{l} \in \mathbb{N}^{m}} \varphi(\boldsymbol{l}) x_{1}\left(\pi_{1}(\boldsymbol{l})\right) \cdots x_{n}\left(\pi_{n}(\boldsymbol{l})\right),
$$

$\left(x_{1}, \ldots, x_{n}\right) \in \mathcal{H}_{1} \times \cdots \times \mathcal{H}_{n}$. The Cauchy-Schwarz inequality implies

Lemma 7.2 ([B1, Lemma 1.2]). $\mathcal{A}_{\varphi}$ is a bounded n-linear form on $\mathcal{H}$ such that

$$
\left\|\mathcal{A}_{\varphi}\right\|:=\sup \left\{\left|\mathcal{A}_{\varphi}\left(x_{1}, \ldots, x_{n}\right)\right|:\left\|x_{i}\right\|_{\mathcal{H}} \leq 1, i \in[n]\right\} \leq\|\varphi\|_{\infty} .
$$

Let $V_{U}\left(\mathbb{N}^{m}\right)$ denote the space of all $\varphi \in l^{\infty}\left(\mathbb{N}^{m}\right)$ which can be written as

$$
\varphi(\boldsymbol{l})=\sum_{i=1}^{\infty} a_{i} \theta_{1, i}\left(\pi_{1}(\boldsymbol{l})\right) \cdots \theta_{n, i}\left(\pi_{n}(\boldsymbol{l})\right),
$$

where $\boldsymbol{l} \in \mathbb{N}^{m}, \theta_{\alpha, i} \in$ unit ball of $l^{\infty}\left(\mathbb{N}^{k_{\alpha}}\right)(i \in \mathbb{N})$, and $\sum\left|a_{i}\right|<\infty$. Norm $V_{U}\left(\mathbb{N}^{m}\right)$ by

$$
\|\varphi\|_{V_{U}}=\inf \left\{\sum_{i}\left|a_{i}\right|: \text { representations of } \varphi \text { by (7.6) }\right\} \text {. }
$$

Let $\widetilde{V}_{U}\left(\mathbb{N}^{m}\right)$ be the algebra of pointwise limits of sequences that are bounded in $V_{U}\left(\mathbb{N}^{m}\right)$. Norm $\widetilde{V}_{U}\left(\mathbb{N}^{m}\right)$ by

$$
\|\varphi\|_{\widetilde{V}_{U}}=\inf \left\{\sup _{j}\left\|\varphi_{j}\right\|_{V_{U}}: \lim _{j \rightarrow \infty} \varphi_{j}(\boldsymbol{l})=\varphi(\boldsymbol{l}), \boldsymbol{l} \in \mathbb{N}^{m}\right\} .
$$

Theorem 7.3 ([B1, Theorem 1.3]). Let $\varphi \in l^{\infty}\left(\mathbb{N}^{m}\right)$, and let $\mathcal{A}_{\varphi}$ be the n-linear form on $\mathcal{H}$ defined by (7.5). Then $\mathcal{A}_{\varphi}$ is projectively bounded if and only if $\varphi \in$ $\widetilde{V}_{U}\left(\mathbb{N}^{m}\right)$.

The statement of Theorem 1.3 in [B1] involved an algebra of restrictions of Fourier-Stieltjes transforms to a "fractional" Cartesian product of a lacunary spectral set. This algebra is canonically isomorphic to $\widetilde{V}_{U}\left(\mathbb{N}^{m}\right)$.

Let $e_{U}$ be the combinatorial dimension of the "fractional" Cartesian product $\left\{\left(\pi_{1}(\boldsymbol{l}), \ldots, \pi_{n}(\boldsymbol{l})\right): \boldsymbol{l} \in \mathbb{N}^{m}\right\}$, which can be computed by solving a linear programming problem (see $[\mathrm{BS}])$. We have $l^{\infty}\left(\mathbb{N}^{m}\right)=\widetilde{V}_{U}\left(\mathbb{N}^{m}\right)$ if and only if $e_{U}=1$ (e.g., [B2, Corollary 2]), and thus obtain

Corollary 7.4. $\left\|\mathcal{A}_{\varphi}\right\|_{p b_{n}}<\infty$ for all $\varphi \in l^{\infty}\left(\mathbb{N}^{m}\right)$ if and only if $e_{U}=1$. 
For example, if $n \geq 2$ and $m=1$ (i.e., $U=\{(1), \ldots,(1)\}$ and $e_{U}=1$ ), then

$$
\mathcal{A}_{U}\left(x_{1}, \ldots, x_{n}\right)=\sum_{l \in \mathbb{N}} \varphi(l) x_{1}(l) \cdots x_{n}(l), \quad x_{1} \in l^{2}, \ldots, x_{n} \in l^{2},
$$

is a projectively bounded $n$-linear form on $l^{2}$ for all $\varphi \in l^{\infty}(\mathbb{N})$ (the instance $n=2$ is the classical Grothendieck inequality). On the other hand, if $n=3, m=3$ and $U=\{(1,2),(2,3),(1,3)\}$, then $e_{U}=3 / 2$. Therefore there exists $\varphi \in l^{\infty}\left(\mathbb{N}^{3}\right)$ such that

$$
\begin{aligned}
\mathcal{A}_{\varphi}(x, y, z)=\sum_{(i, j, k) \in \mathbb{N}^{3}} \varphi(i, j, k) x(i, j) y(j, k) z(i, k), & \\
x & \in l^{2}\left(\mathbb{N}^{2}\right), \quad y \in l^{2}\left(\mathbb{N}^{2}\right), \quad z \in l^{2}\left(\mathbb{N}^{2}\right),
\end{aligned}
$$

is a projectively unbounded form (such $\mathcal{A}_{\varphi}$ appeared first in [V3]).

The multilinear forms defined in (7.5) naturally give rise to $\mathcal{F}$-measures in the classical framework of the circle group $[0,2 \pi):=\mathbf{T}$. For each $\alpha \in[n]$, fix a $k_{\alpha^{-}}$ dimensional enumeration of $\mathbb{Z}(=\widehat{\mathbf{T}})$ which we write as $\mathbb{Z}=\left\{e_{\mathbf{j}}: \mathbf{j} \in \mathbb{N}^{k_{\alpha}}\right\}$ (cf. (7.3)). Let $\mathfrak{B}$ denote the Borel field of $\mathbf{T}$. For each $\varphi \in l^{\infty}\left(\mathbb{N}^{m}\right)$, define

$$
\mu_{\varphi}\left(E_{1}, \ldots, E_{n}\right)=\sum_{\boldsymbol{l} \in \mathbb{N}^{m}} \varphi(\boldsymbol{l}) \hat{\mathbf{1}}_{E_{1}}\left(e_{\pi_{1}(\boldsymbol{l})}\right) \cdots \hat{\mathbf{1}}_{E_{n}}\left(e_{\pi_{n}(\boldsymbol{l})}\right),
$$

$E_{1} \in \mathfrak{B}, \ldots, E_{n} \subset \mathfrak{B}$. Plancherel's theorem and Lemma 7.2 imply that $\mu_{\varphi} \in$ $\mathcal{F}_{n}\left(\mathfrak{B}^{n}\right)$ and

$$
\left\|\mu_{\varphi}\right\|_{\mathcal{F}_{n}} \leq\|\varphi\|_{\infty}
$$

Specifically, if $f_{1} \in L^{2}(\mathbf{T}, d x), \ldots, f_{n} \in L^{2}(\mathbf{T}, d x)(d x=$ Lebesgue measure on $\mathfrak{B})$, then

$$
\int f_{1} \otimes \cdots \otimes f_{n} d \mu_{\varphi}:=\mathcal{A}_{\varphi}\left(\hat{f}_{1}, \ldots, \hat{f}_{n}\right)
$$

and

$$
\left|\int f_{1} \otimes \cdots \otimes f_{n} d \mu_{\varphi}\right| \leq\|\varphi\|_{\infty}\left\|f_{1}\right\|_{2} \cdots\left\|f_{n}\right\|_{2} .
$$

Theorem 7.5. Suppose $\varphi \in l^{\infty}\left(\mathbb{N}^{n}\right)$, and let $\mu_{\varphi} \in \mathcal{F}_{n}\left(\mathfrak{B}^{n}\right)$ be defined by (7.10). Then, $\mu_{\varphi} \in \mathcal{P B} \mathcal{F}_{n}\left(\mathfrak{B}^{n}\right)$ if and only if $\varphi \in \widetilde{V}_{U}\left(\mathbb{N}^{n}\right)$. In particular, $\mu_{\varphi} \in \mathcal{P B \mathcal { F }}\left(\mathfrak{B}^{n}\right)$ for all $\varphi \in l^{\infty}\left(\mathbb{N}^{n}\right)$ if and only if $e_{U}=1$.

Proof. Following (7.12), the if direction follows from the if direction of Theorem 7.3.

To obtain the only if direction, first note, again by (7.12), that if $\mathbf{j}_{1} \in \mathbb{N}^{k_{1}}, \ldots$, $\mathbf{j}_{n} \in \mathbb{N}^{k_{n}}$, then

$$
\hat{\mu}_{\varphi}\left(e_{\mathbf{j}_{1}}, \ldots, e_{\mathbf{j}_{n}}\right)= \begin{cases}\varphi(\boldsymbol{l}), & \text { if } \pi_{1}(\boldsymbol{l})=\mathbf{j}_{1}, \ldots, \pi_{n}(\boldsymbol{l})=\mathbf{j}_{n}, \\ 0, & \text { otherwise. }\end{cases}
$$

Now apply Proposition 4.1. 


\section{ANOTHER CHARACTERIZATION}

We continue in the setting of the previous section. In addition to the $k_{\alpha^{-}}$ dimensional enumerations of $\mathbb{Z}(\alpha \in[n])$, we fix also an $m$-dimensional enumeration $\mathbb{Z}=\left\{e_{\boldsymbol{l}}: \boldsymbol{l} \in \mathbb{N}^{m}\right\}$. If $\varphi \in l^{\infty}\left(\mathbb{N}^{m}\right)$, then

$$
\lambda_{\varphi}\left(E_{1}, \ldots, E_{n}, C\right)=\sum_{\boldsymbol{l} \in \mathbb{N}^{m}} \varphi(\boldsymbol{l}) \hat{\mathbf{1}}_{E_{1}}\left(e_{\pi_{1}(\boldsymbol{l})}\right) \cdots \hat{\mathbf{1}}_{E_{n}}\left(e_{\pi_{n}(\boldsymbol{l})}\right) \mathbf{1}_{C}\left(e_{\boldsymbol{l}}\right),
$$

$E_{1} \times \cdots \times E_{n} \in \mathfrak{B}^{n}, C \subset \mathbb{Z}$, determines an $\mathcal{F}_{n+1}$-measure on $\mathfrak{B}^{n} \times \mathbb{Z}$ such that

$$
\left\|\lambda_{\varphi}\right\|_{\mathcal{F}_{n+1}} \leq\|\varphi\|_{\infty}
$$

(cf. Lemma 7.2). Moreover, if $f_{1} \in L^{2}(\mathbf{T}, d x), \ldots, f_{n} \in L^{2}(\mathbf{T}, d x)$, and $g \in l^{\infty}\left(\mathbb{N}^{m}\right)$, then

$$
\int f_{1} \otimes \cdots \otimes f_{n} \otimes g d \lambda_{\varphi}=\sum_{\boldsymbol{l} \in \mathbb{N}^{m}} \varphi(\boldsymbol{l}) \hat{f}_{1}\left(e_{\pi_{1}(\boldsymbol{l})}\right) \cdots \hat{f}_{n}\left(e_{\pi_{n}(\boldsymbol{l})}\right) g\left(e_{\boldsymbol{l}}\right) .
$$

Observe that $\mu$ in the proof of Theorem 6.1 was synthesized from $\mathcal{F}_{3}$-measures given essentially by (8.1), where $n=2, U=\{(1),(1)\}$, and $\varphi \equiv 1$ (see (6.5) and (6.6)).

Theorem 8.1. Suppose $\varphi \in l^{\infty}\left(\mathbb{N}^{n}\right)$, and let $\lambda_{\varphi} \in \mathcal{F}_{n+1}\left(\mathfrak{B}^{n} \times \mathbb{Z}\right)$ be defined by (8.1). Then, $\lambda_{\varphi} \in \mathcal{P B \mathcal { F }}{ }_{n+1}\left(\mathfrak{B}^{n} \times \mathbb{Z}\right)$ if and only if $\varphi \in l^{2}\left(\mathbb{N}^{m}\right)$.

Proof. To verify the only if direction, we can be assume that $\varphi$ has finite support, which we denote by $D$. Suppose $|D|=N$, and identify $D$ with $\mathbb{Z}_{N}$. Designate this identification by $\boldsymbol{l} \leftrightarrow d_{\boldsymbol{l}}, \boldsymbol{l} \in D, d_{\boldsymbol{l}} \in \mathbb{Z}_{N}$, and then view $\lambda_{\varphi}$ as an $\mathcal{F}_{n+1^{-}}$-measure on $\mathfrak{B}^{n} \times \mathbb{Z}_{N}$. If $\mathbf{j}_{1} \in \mathbb{N}^{k_{1}}, \ldots, \mathbf{j}_{n} \in \mathbb{N}^{k_{n}}, \hat{j} \in \widehat{\mathbb{Z}}_{N}$, then

$$
\hat{\lambda}_{\varphi}\left(e_{\mathbf{j}_{1}}, \ldots, e_{\mathbf{j}_{n}}, \hat{j}\right)= \begin{cases}\varphi(\boldsymbol{l}) \psi_{N}\left(\hat{j}, d_{\boldsymbol{l}}\right) & \text { if } \pi_{1}(\boldsymbol{l})=\mathbf{j}_{1}, \ldots, \pi_{n}(\boldsymbol{l})=\mathbf{j}_{n}, \boldsymbol{l} \in D, \\ 0, & \text { otherwise }\end{cases}
$$

(see (6.1) for the definition of $\left.\psi_{N}\right)$. By assumption,

$$
\left\|\hat{\lambda}_{\varphi}\right\|_{V_{n+1}\left(\mathbb{Z}^{n} \times \widehat{\mathbb{Z}}_{N}\right)} \leq\left\|\lambda_{\varphi}\right\|_{\mathcal{P B F}_{n+1}}<\infty .
$$

Let $\theta$ be in the unit ball of $l^{2}(D)$. Consider

$$
\left|\sum_{\boldsymbol{l} \in D} \varphi(\boldsymbol{l}) \theta(\boldsymbol{l})\right|=\left|\sum_{\boldsymbol{l} \in D} \sum_{\hat{j} \in \widehat{\mathbb{Z}}_{N}} \varphi(\boldsymbol{l}) \psi_{N}\left(\hat{j}, f_{\boldsymbol{l}}\right)\left(\frac{1}{N}\right) \theta(\boldsymbol{l}) \overline{\psi_{N}\left(\hat{j}, d_{\boldsymbol{l}}\right)}\right|,
$$

which represents the dual action between $\hat{\lambda}_{\varphi}$ and $(1 / N) \hat{\lambda}_{\theta}$. Observe that

$$
\left\|\left(\frac{1}{N}\right) \hat{\lambda}_{\theta}\right\|_{\mathcal{F}_{n+1}} \leq 1
$$

(to verify (8.7), use an argument similar to the one establishing that (6.2) implies (6.3)). Now apply (8.5) and (8.7) to (8.6), maximize over $\theta$, and deduce $\|\varphi\|_{2} \leq$ $\left\|\lambda_{\varphi}\right\|_{\mathcal{P B F}_{n+1}}$.

Conversely, assume that $\varphi \in l^{2}\left(\mathbb{N}^{m}\right)$. In the statement of Theorem 7.3 replace $n$ by $n+1$ and $U$ by $U \cup\{[m]\}$; let $\varphi \equiv 1$, and denote the corresponding $(n+1)$-linear form by $\mathcal{A}$. Then, (8.3) can be rewritten as

$$
\int f_{1} \otimes \cdots \otimes f_{n} \otimes g d \lambda_{\varphi}=\mathcal{A}\left(\hat{f}_{1}, \ldots, \hat{f}_{n}, \varphi g\right) .
$$

By assumption, $\varphi g \in l^{2}\left(\mathbb{N}^{m}\right)$ for all $g \in l^{\infty}\left(\mathbb{N}^{m}\right)$. Therefore, by Theorem 7.3, $\|\mathcal{A}\|_{p b_{n}}<\infty$, and this, by (8.8), implies $\left\|\lambda_{\varphi}\right\|_{p b_{n}}<\infty$. 


\section{Applications to stochastic analysis}

i. Random multilinear forms. Let $X=\left\{X_{j}: j \in \mathbb{N}\right\}$ be a system of random variables on a probability space $(\Omega, \mathfrak{A}, \mathbb{P})$. Define

$$
\|X\|_{(1)}=\sup \left\{\left\|\sum_{j=1}^{m} \epsilon_{j} X_{j}\right\|_{L^{1}(\Omega, \mathbb{P})}: \epsilon_{j}= \pm 1, m \in \mathbb{N}, j \in \mathbb{N}\right\} .
$$

For $A \in \mathfrak{A}$ and finite subsets of $B$ of $\mathbb{N}$, define

$$
\mu_{X}(A, B)=\sum_{j \in B} \mathbf{E} \mathbf{1}_{A} X_{j}
$$

(E denotes expectation).

Lemma 9.1. Let $X=\left\{X_{j}: j \in \mathbb{N}\right\}$ be a system of random variables such that $\|X\|_{(1)}<\infty$. Then:

(i) $\mu_{X} \in \mathcal{F}_{2}(\mathfrak{A} \times \mathbb{N})$, and $\left\|\mu_{X}\right\|_{\mathcal{F}_{2}}=\|X\|_{(1)}$.

(ii) There exists a probability measure $\nu$ on $\mathbb{N}$ such that, for all $b \in L^{2}(\mathbb{N}, \nu)$, $\sum_{j=1}^{\infty} b_{j} X_{j}$ converges in $L^{1}(\Omega, \mathbb{P})$, and

$$
\left\|\sum_{j=1}^{\infty} b_{j} X_{j}\right\|_{L^{1}(\Omega, \mathbb{P})} \leq \mathcal{K}_{\mathcal{G}}\|b\|_{L^{2}(\nu)}\|X\|_{(1)} .
$$

Proof. (i) Let $a \mathbb{N}$ denote the algebra generated by the finite subsets of $\mathbb{N}$. The definition of $\|X\|_{(1)}$ implies that $\mu_{X}$ is an $\mathcal{F}_{2}$-measure on $\mathfrak{A} \times a \mathbb{N}$ and $\left\|\mu_{X}\right\|_{\mathcal{F}_{2}(\mathfrak{A} \times a \mathbb{N})}=$ $\|X\|_{(1)}$. Now apply Theorem 2.2 .

(iii) The map $(Y, b) \rightarrow \int b \otimes Y d \mu_{X}$ is a bounded bilinear functional on $L^{\infty}(\Omega, \mathbb{P}) \times$ $c_{0}(\mathbb{N})$. View $L^{\infty}(\Omega, \mathbb{P})$ as a space of continuous functions on the maximal ideal space of $L^{\infty}(\Omega, \mathbb{P})$. Then apply Theorem 2.5(ii) to obtain a probability measure $\nu$ on $\mathbb{N}$ such that for every $Y \in L^{\infty}(\Omega, \mathbb{P})$ and $b \in c_{0}(\mathbb{N})$,

$$
\left|\int b \otimes Y d \mu_{X}\right| \leq \mathcal{K}_{\mathcal{G}}\|b\|_{L^{2}(\nu)}\|Y\|_{L^{\infty}(\Omega, \mathbb{P})}\|X\|_{(1)} .
$$

Since $\int b \otimes Y d \mu_{X}=\mathbf{E} Y \sum_{j \in \mathbb{N}} b_{j} X_{j},(9.4)$ implies that for $m>k>0$

$$
\left|\mathbf{E} Y \sum_{j=k}^{m} b_{j} X_{j}\right|<\mathcal{K}_{\mathcal{G}}\|b\|_{\infty} \nu(\{k, \ldots, m\})^{1 / 2}\|Y\|_{L^{\infty}(\Omega, \mathbb{P})}\|X\|_{(1)} .
$$

This implies the assertion.

Let $X^{(i)}=\left\{X_{j}^{(i)}: j \in \mathbb{N}\right\}, i=1, \ldots, n$, be mutually independent systems such that $\left\|X^{(i)}\right\|_{(1)}<\infty$. The $X^{(i)}$ 's can be redefined on $\left(\Omega^{n}, \sigma\left(\mathfrak{A}^{n}\right), \mathbb{P}^{n}\right)$ so that each $X^{(i)}$ depends only on the $i$ th coordinate $(i=1, \ldots, n)$. For $\left(A_{1}, \ldots, A_{n}\right) \in \mathfrak{A}^{n}$ and 
$B_{1} \times \cdots \times B_{n} \subset \mathbb{N}^{n}$, define

$$
\begin{aligned}
\mu_{X^{(1)}} \otimes \cdots \otimes X^{(n)} & \left(\left(A_{1}, \ldots, A_{n}\right),\left(B_{1}, \ldots, B_{n}\right)\right) \\
& :=\sum_{j_{1} \in B_{1}, \ldots, j_{n} \in B_{n}} \mathbf{E} \mathbf{1}_{A_{1}} \cdots \mathbf{1}_{A_{n}} X_{j_{1}}^{(1)} \cdots X_{j_{n}}^{(n)} \\
& =\left(\sum_{j \in B_{1}} \mathbf{E} \mathbf{1}_{A_{1}} X_{j}^{(1)}\right) \cdots\left(\sum_{j \in B_{n}} \mathbf{E} \mathbf{1}_{A_{n}} X_{j}^{(n)}\right) \\
& =\mu_{X^{(1)}}\left(A_{1}, B_{1}\right) \cdots \mu_{X^{(n)}}\left(A_{n}, B_{n}\right) .
\end{aligned}
$$

Theorem 9.2. Let $X^{(i)}(i=1, \ldots, n)$ be as above. Then:

(i) $\mu_{X^{(1)} \otimes \cdots \otimes X^{(n)}} \in \mathcal{F}_{2}\left(\sigma\left(\mathfrak{A}^{n}\right), \mathbb{N}^{n}\right)$ and

$$
\left\|\mu_{X^{(1)} \otimes \cdots \otimes X^{(n)}}\right\|_{\mathcal{F}_{2}} \leq\left(\mathcal{K}_{\mathcal{G}}^{2}\right)^{n-1}\left\|X^{(1)}\right\|_{(1)} \cdots\left\|X^{(n)}\right\|_{(1)} ;
$$

(ii) there exists a probability measure $\nu$ on $\mathbb{N}^{n}$ such that for all $b \in L^{2}\left(\mathbb{N}^{n}, \nu\right)$ the series

$$
\sum_{\left(j_{1}, \ldots, j_{n}\right) \in \mathbb{N}^{n}} b_{j_{1} \cdots j_{n}} X_{j_{1}}^{(1)} \cdots X_{j_{n}}^{(n)}
$$

converges in $L^{1}(\Omega, \mathbb{P})$, and

$\left\|\sum_{\left(j_{1}, \ldots, j_{n}\right) \in \mathbb{N}^{n}} b_{j_{1} \cdots j_{n}} X_{j_{1}}^{(1)} \cdots X_{j_{n}}^{(n)}\right\|_{L^{1}\left(\Omega^{n}, \mathbb{P}^{n}\right)} \leq \mathcal{K}_{\mathcal{G}}^{2 n-1}\|b\|_{L^{2}(\nu)}\left\|X^{(1)}\right\|_{(1)} \cdots\left\|X^{(n)}\right\|_{(1)}$.

Proof. (i) By induction, Lemma 9.1 and Theorems 5.1 and 1.3 imply that $\mu_{X_{(1)} \otimes \cdots \otimes X^{(n)}}$ determines an $\mathcal{F}_{2}$-measure on $\sigma\left(\mathfrak{A}^{n}\right) \times \mathbb{N}^{n}$. (9.7) follows from Corollary 3.3.

(ii) By part (i), $\mu_{X^{(1)} \otimes \cdots \otimes X^{(n)}}$ determines a bounded bilinear functional on $L^{\infty}\left(\Omega^{n}, \mathbb{P}^{n}\right) \times c_{0}\left(\mathbb{N}^{n}\right)$. Then, as in the proof of Lemma 9.1(ii), an application of Theorem 2.5(ii) implies the assertion.

Remarks. 1. A review of the proof of Theorem 5.1 yields that the probability measure $\nu$ in Theorem 9.4 can be taken to be $\nu_{1} \times \cdots \times \nu_{n}$, where each of the $\nu_{i}$ 's is the probability measure in Lemma 9.1(ii).

2. Decoupling inequalities (e.g., see $[\mathrm{McT}]$ ) imply

Corollary 9.3. Let $X=\left\{X_{j}: j \in \mathbb{N}\right\}$ be a system of independent symmetric random variables such that

$$
\sup \left\{\left\|\sum_{j=1}^{m} X_{j}\right\|_{L^{1}}: m \in \mathbb{N}\right\} \quad\left(=\|X\|_{(1)}\right)<\infty .
$$

Let $W_{n}$ denote $\left\{\left(j_{1}, \ldots, j_{n}\right): 1 \leq j_{1}<\cdots<j_{n}<\infty\right\}$. Then, there exists a probability measure $\nu$ on $W_{n}$ such that for all $b \in L^{2}\left(W_{n}, \nu\right)$ the series

$$
\sum_{\left(j_{1}, \ldots, j_{n}\right) \in W_{n}} b_{j_{1} \cdots j_{n}} X_{j_{1}} \cdots X_{j_{n}}
$$


converges in $L^{1}(\Omega, \mathbb{P})$, and

$$
\left\|\sum_{\left(j_{1}, \ldots, j_{n}\right) \in W_{n}} b_{j_{1} \cdots j_{n}} X_{j_{1}} \cdots X_{j_{n}}\right\|_{L^{1}\left(\Omega^{n}, \mathbb{P}^{n}\right)} \leq \mathcal{K}_{\mathcal{G}}^{2 n-1}\|b\|_{L^{2}(\nu)}\|X\|_{(1)} .
$$

E. Giné pointed out to me that if each $X^{(i)}$ in Theorem 9.2 is a system of independent symmetric random variables, then the assertions in part (i) and part (ii) with $l^{\infty}\left(\mathbb{N}^{n}\right)$ in place of $L^{2}\left(\mathbb{N}^{n}, \nu\right)$ can be proved by a use of Khintchin's inequality. Even this instance of Theorem 9.2 appears to have been overlooked by workers in the area (cf. [KW, Chapter 6]).

ii. Stochastic integration (cf. [B6]). Let $X=\left\{X(\mathbf{t}): \mathbf{t} \in[0,1]^{n}\right\}$ be an $n$ process on $(\Omega, \mathfrak{A}, \mathbb{P})$ (a process indexed by $n$ parameters), and define

$$
\tilde{\mu}_{X}(A, \mathbf{I})=\mathbf{E} \mathbf{1}_{A} \Delta^{n} X(\mathbf{I}), \quad A \in \mathfrak{A}, \mathbf{I}=\left(s_{1}, t_{1}\right] \times \cdots \times\left(s_{n}, t_{n}\right] \subset[0,1]^{n}
$$

$\left(\Delta^{n}\right.$ denotes the operation of taking $n$th order differences). Let $\mathfrak{O}$ be the algebra generated by $\{(s, t]: 0 \leq s<t \leq 1\}$, and extend the domain of $\tilde{\mu}_{X}$ by finite additivity to $\mathfrak{O}^{n}$. Assume that $\left\|\tilde{\mu}_{X}\right\|_{\mathcal{F}_{n+1}\left(\mathfrak{A} \times \mathfrak{O}^{n}\right)}<\infty$; we shall refer to such $X$ as an $n$-integrator. Then, for every $A \in \mathfrak{A}$, the function $\mathbf{E} \mathbf{1}_{A} X(\mathbf{u})\left(\mathbf{u} \in[0,1]^{n}\right)$ has bounded variation in the sense of Fréchet (e.g., [MT]), and therefore we can define $X^{+}=\left\{X^{+}(\mathbf{t}): \mathbf{t} \in[0,1]^{n}\right\}$ by

$$
\mathbf{E} \mathbf{1}_{A} X^{+}(\mathbf{t})=\lim _{\mathbf{u} \rightarrow \mathbf{t}^{+}} \mathbf{E} \mathbf{1}_{A} X(\mathbf{u}), \quad A \in \mathfrak{A}, \mathbf{t} \in[0,1]^{n}
$$

$\left(\lim _{\mathbf{u} \rightarrow \mathbf{t}^{+}}\right.$denotes right limits along coordinate axes; e.g., [MT, Theorem 2.2]) Define

$$
\mu_{X}(A, \mathbf{I})=\tilde{\mu}_{X^{+}}(A, \mathbf{I}), \quad A \in \mathfrak{A} \text { and } \mathbf{I} \in \mathfrak{O}^{n},
$$

and deduce, from Theorem 2.2, that $\mu_{X}$ determines an $\mathcal{F}_{n+1}$-measure on $\mathfrak{A} \times \mathfrak{B}^{n}$, where $\mathfrak{B}$ denotes the Borel field in $[0,1]$. If $\left\|\mu_{X}\right\|_{p b_{n}}<\infty$, then $X$ is said to be projectively bounded.

If $X$ and $Y$ are $n$-processes, then $X \otimes Y$ will denote the $2 n$-process defined by $X \otimes Y(\mathbf{s}, \mathbf{t})=X(\mathbf{s}) Y(\mathbf{t}),(\mathbf{s}, \mathbf{t}) \in[0,1]^{n} \times[0,1]^{n}$. Theorem 1.3 implies

Theorem 9.4. If $X$ and $Y$ are mutually independent $n$-integrators (i.e., $X(\mathbf{s})$ and $Y(\mathbf{t})$ are independent random variables for all $\left.(\mathbf{s}, \mathbf{t}) \in[0,1]^{n} \times[0,1]^{n}\right)$ and $X$ is projectively bounded, then

$$
\mu_{X \otimes Y} \in \mathcal{F}_{n+1}\left(\mathfrak{A} \times \sigma\left(\mathfrak{B}^{2}\right) \times \cdots \times \sigma\left(\mathfrak{B}^{2}\right)\right),
$$

and

$$
\mu_{X \otimes Y}=\mu_{X} \times \mu_{Y}
$$

Remarks. 1. Denote $\mathbf{I}_{\mathbf{t}}=\left(0, t_{1}\right] \times \cdots \times\left(0, t_{n}\right], \mathbf{t}=\left(t_{1}, \ldots, t_{n}\right) \in[0,1]^{n}$. Suppose $X$ is a projectively bounded $n$-process, and $Y$ is an $n$-integrator independent of $X$. Then, by Theorem 9.4 and Proposition 2.3, $\int \mathbf{1}_{\mathbf{I}_{\mathbf{t}} \cap \mathbf{I}_{\mathbf{u}}}(\mathbf{s}) \mu_{X} \times \mu_{Y}((d \mathbf{s}, \cdot),(d \mathbf{u}, \cdot))$ determines a scalar measure on $(\Omega, \mathfrak{A})$ which is absolutely continuous with respect to $\mathbb{P}$. We can then define the integral $\int X d Y$ (or $\int Y d X$ ) to be the $n$-process given by

$$
\left(\int X d Y\right)(\mathbf{t}):=\frac{d}{d \mathbb{P}}\left(\int \mathbf{1}_{\mathbf{I}_{\mathbf{t}} \cap \mathbf{I}_{\mathbf{u}}}(\mathbf{s}) \mu_{X} \times \mu_{Y}((d \mathbf{s}, \cdot) \times(d \mathbf{u}, \cdot))\right) .
$$


To obtain the integral of $Y$ with respect to $X$, merely interchange the roles of $X$ and $Y$. It is evident that $\int X d Y$ is an $n$-integrator. An integration by parts formula is immediate:

$$
\left(\int X d Y\right)(\mathbf{t})=\Delta^{n} X^{+}\left(\mathbf{I}_{\mathbf{t}}\right) \Delta^{n} Y^{+}\left(\mathbf{I}_{\mathbf{t}}\right)-\left(\int Y d X\right)(\mathbf{t}) .
$$

2. An inductive application of Theorems 5.1 and 9.4 implies that if $X^{(1)}, \ldots, X^{(n)}$ are mutually independent 1-integrators, and $f$ is a bounded measurable function on $[0,1]^{n}$, then we can define the stochastic integral

$$
\begin{aligned}
\int_{0}^{1} \cdots & \int_{0}^{1} f\left(t_{1}, \ldots, t_{n}\right) X^{(1)}\left(d t_{1}\right) \cdots X^{(n)}\left(d t_{n}\right) \\
& :=\frac{d}{d \mathbb{P}}\left(\int_{[0,1]^{n}} f(\mathbf{t}) \mu_{X^{(1)}} \times \cdots \times \mu_{X^{(n)}}(\cdot, d \mathbf{t})\right) .
\end{aligned}
$$

Then, Theorem 2.5(ii) implies that there exists a probability measure $\nu$ on $[0,1]^{n}$ such that the integral above can be defined for all $f \in L^{2}\left([0,1]^{n}, \nu\right)$ (cf. [B6, Corollary 3.2$])$.

3. One of the most widely studied stochastic integrals over one-dimensional parameter sets has been the Itô integral (e.g. $[\mathrm{Pr}])$. In it, a random integrand is adapted to a 1-integrator which is a semi-martingale $\left(=L^{2}\right.$-bounded martingale + finite variation process; loosely put, an integrand is adapted if it is a function of the respective integrator). In a general framework, not every 1-integrator is a semimartingale and not every prospective integrand is adapted. Since every 1-integrator is projectively bounded (Theorem 5.1), every 1-integrator is integrable (in the sense of (9.13)) with respect to any other that is independent of it. Independence is the opposite of adaptability; how to deal effectively with random integrands that may be neither independent nor functions of the respective integrators is an open-ended question.

4. An example of a 1-integrator is a 1-process $X$ with independent symmetric increments such that $\mathbf{E}|X(1)|<\infty$. For $n>1$, examples of $n$-integrators $X$, projectively bounded or unbounded, with the property that $\mu_{X}$ cannot be extended to $\mathcal{F}_{n-1}$-measures, can be obtained from the $\mathcal{F}$-measures construction in sections 7 and 8 .

\section{Projective Boundedness, $L^{2}$-Factorizability, COMPLETE BOUNDEDNESS, AND CONVOLVABILITY}

i. Proposition 2.1 implies that for all $n \geq 1$

$$
\mathcal{F}_{1}\left(\sigma\left(\mathfrak{A}_{1} \times \cdots \times \mathfrak{A}_{n}\right)\right) \subset \mathcal{P} \mathcal{B F}_{n}\left(\mathfrak{A}_{1} \times \cdots \times \mathfrak{A}_{n}\right) .
$$

In this paper we noted that $\mathcal{F}_{2}\left(\mathfrak{A}_{1} \times \mathfrak{A}_{2}\right) \subset \mathcal{P} \mathcal{B F}_{2}\left(\mathfrak{A}_{1} \times \mathfrak{A}_{2}\right)$ (Theorem 5.1), and that for every $n>2$ there exist $\mathcal{F}_{n}$-measures which are not projectively bounded (Theorem 6.1). In this light, it is natural to ask whether, for all $n \geq 2$,

$$
\mathcal{F}_{2}\left(\sigma\left(\mathfrak{A}_{1} \times \cdots \times \mathfrak{A}_{n-1}\right) \times \mathfrak{A}_{n}\right) \subset \mathcal{P} \mathcal{B} \mathcal{F}_{n}\left(\mathfrak{A}_{1} \times \cdots \times \mathfrak{A}_{n}\right) ?
$$


The answer is no: Consider the class of $\mathcal{F}_{4}$-measures given by (7.10) in the case $m=2$ and $U=\{(1),(2),(1),(2)\}$. That is, let $\varphi \in l^{\infty}\left(\mathbb{N}^{2}\right)$ and define

$$
\mu_{\varphi}(A, B, C, D)=\sum_{(j, k) \in \mathbb{Z}^{2}} \varphi(j, k) \hat{\mathbf{1}}_{A}(j) \hat{\mathbf{1}}_{B}(k) \hat{\mathbf{1}}_{C}(j) \hat{\mathbf{1}}_{D}(k), \quad(A, B, C, D) \in \mathfrak{B}^{4} .
$$

Such $\mu_{\varphi}$ is extendible to the $\mathcal{F}_{2}$-measure on $\sigma\left(\mathfrak{B}^{2}\right) \times \sigma\left(\mathfrak{B}^{2}\right)$ defined by

$$
\sum_{(j, k) \in \mathbb{Z}^{2}} \varphi(j, k) \hat{\mathbf{1}}_{A}(j, k) \hat{\mathbf{1}}_{B}(j, k), \quad A \in \sigma\left(\mathfrak{B}^{2}\right), B \in \sigma\left(\mathfrak{B}^{2}\right) .
$$

However, there exist $\varphi \in l^{2}\left(\mathbb{N}^{2}\right)$ such that $\varphi \notin V_{U}\left(\mathbb{N}^{2}\right)\left(e_{U}=2\right.$; see the comment following Theorem 7.3), and thus, by Theorem 7.5, $\mu_{\varphi} \notin \mathcal{P} \mathcal{B F}_{4}\left(\mathfrak{B}^{4}\right)$.

It can be verified that if any one of the $\mathcal{F}_{3}$-measures given either by (7.10) or (8.1) is extendible to an $\mathcal{F}_{2}$-measure, then it is necessarily in $\mathcal{P} \mathcal{B} \mathcal{F}_{3}$. I do not know the answer to (10.2) in the case $n=3$.

In subsections ii and iii, below, $\mathcal{X}_{1}, \ldots, \mathcal{X}_{n}$ denote locally compact Hausdorff spaces with respective Borel fields $\mathfrak{B}_{1}, \ldots, \mathfrak{B}_{n}$.

ii. A bounded $n$-linear functional $\mu$ on $\mathcal{C}_{0}\left(\mathcal{X}_{1}\right) \times \cdots \times \mathcal{C}_{0}\left(\mathcal{X}_{n}\right)$ (that is,

$$
\left.\mu \in \mathcal{F}_{n}\left(\mathfrak{B}_{1} \times \cdots \times \mathfrak{B}_{n}\right)\right)
$$

is said to be $L^{2}$-factorizable if there exist $\mathcal{K}>0$ and probability measures $\nu_{1}, \ldots, \nu_{n}$ on $\mathfrak{B}_{1}, \ldots, \mathfrak{B}_{n}$, respectively, such that for all $f_{1} \in \mathcal{C}_{0}\left(\mathcal{X}_{1}\right), \ldots, f_{n} \in \mathcal{C}_{0}\left(\mathcal{X}_{n}\right)$,

$$
\left|\int f_{1} \otimes \cdots \otimes f_{n} d \mu\right| \leq \mathcal{K}\left\|f_{1}\right\|_{L^{2}\left(\nu_{1}\right)} \cdots\left\|f_{n}\right\|_{L^{2}\left(\nu_{n}\right)}
$$

(cf. [B5]). Every bounded linear functional $\mu$ on $\mathcal{C}_{0}\left(\mathcal{X}_{1}\right) \times \mathcal{C}_{0}\left(\mathcal{X}_{2}\right)$ is projectively bounded essentially because every such $\mu$ is $L^{2}$-factorizable. To wit, the argument establishing Theorem 5.1 used Grothendieck's factorization theorem and inequality, and in a two-dimensional setting these two statements are equivalent (e.g., see the Appendix). In higher dimensions, $L^{2}$-factorizability alone will not imply projective boundedness. Indeed, every $\mu_{\varphi}$ given by (7.10) is $L^{2}$-factorizable, but not every such $\mu_{\varphi}$ is projectively bounded (Theorem 7.5). Within the class of $\mathcal{F}$-measures given by (8.1), on the other hand, projective boundedness is equivalent to $L^{2}$-factorizability.

Question 10.1. Is every projectively bounded $\mathcal{F}_{n}$-measure on $\mathfrak{B}_{1} \times \cdots \times \mathfrak{B}_{n}$ necessarily $L^{2}$-factorizable?

iii. An $\mathcal{F}_{n}$-measure on $\mathfrak{B}_{1} \times \cdots \times \mathfrak{B}_{n}$ is said to be completely bounded if there exist a Hilbert space $\mathcal{H}$, ${ }^{*}$-representations $\pi_{i}: C_{0}\left(\mathcal{X}_{i}\right) \rightarrow \mathcal{B}(\mathcal{H}), i=1, \ldots, n$, and vectors $\mathbf{x} \in \mathcal{H}, \mathbf{y} \in \mathcal{H}$ such that for $f_{1} \in \mathcal{C}_{0}\left(\mathcal{X}_{1}\right), \ldots, f_{n} \in \mathcal{C}_{0}\left(\mathcal{X}_{n}\right)$,

$$
\int f_{1} \otimes \cdots \otimes f_{n} d \mu=\left\langle\pi_{1}\left(f_{1}\right) \cdots \pi_{n}\left(f_{n}\right) \mathbf{x}, \mathbf{y}\right\rangle
$$

(see [CES, Corollary 3.2]). Every $\mathcal{F}_{n}$-measure on $\mathfrak{B}_{1} \times \cdots \times \mathfrak{B}_{n}$ which is $L^{2}$-factorizable is also completely bounded, but if $n>2$ then there exists $\mu \in$ $\mathcal{F}_{n}\left(\mathfrak{B}_{1} \times \cdots \times \mathfrak{B}_{n}\right)$ which is completely bounded but not $L^{2}$-factorizable [S]. Since every $\mathcal{F}$-measure given by $(7.10)$ is $L^{2}$-factorizable, Theorem 7.5 implies that there exist $\mathcal{F}$-measures that are completely bounded but not projectively bounded. Moreover, as in the case of $L^{2}$-factorizability, it can be shown that if $\mu$ is an $\mathcal{F}$-measure defined by (8.1), then $\mu$ is projectively bounded if and only if it is completely bounded. 
Question 10.2. Is every $\mu \in \mathcal{P} \mathcal{B F}_{n}\left(\mathfrak{B}_{1} \times \cdots \times \mathfrak{B}_{n}\right)$ completely bounded?

In subsections iv and $\mathrm{v}$, below, $\mathcal{X}_{1}, \ldots, \mathcal{X}_{n}$ denote locally compact Abelian groups with Borel fields $\mathfrak{B}_{1}, \ldots, \mathfrak{B}_{n}$.

iv. In a setting of harmonic analysis, possibly the most important problem at this juncture is whether projective boundedness of $\mu \in \mathcal{F}_{n}$ can be characterized in terms of some general property of $\hat{\mu}$.

Question 10.3. Does the converse to Proposition 4.1 hold? That is, for

$$
\mu \in \mathcal{F}_{n}\left(\mathfrak{B}_{1} \times \cdots \times \mathfrak{B}_{n}\right)
$$

is it true that $\hat{\mu} \in \widetilde{V}_{n}\left(\widehat{\mathcal{X}}_{1} \times \cdots \times \widehat{\mathcal{X}}_{n}\right)$ only if $\mu \in \mathcal{P} \mathcal{B F} \mathcal{F}_{n}\left(\mathfrak{B}_{1} \times \cdots \times \mathfrak{B}_{n}\right)$ ?

An affirmative answer would subsume Theorems 7.5 and 8.1.

v. Suppose $\mu$ and $\varphi$ are $\mathcal{F}_{n}$-measures on $\mathfrak{B}_{1} \times \cdots \times \mathfrak{B}_{n}$. If there exists $\lambda \in$ $\mathcal{F}_{n}\left(\mathfrak{B}_{1} \times \cdots \times \mathfrak{B}_{n}\right)$ such that $\hat{\lambda}=\hat{\mu} \hat{\nu}$ (see (4.1)), then denote $\lambda=\mu \star \nu$. We shall say that $\mu \in \mathcal{F}_{n}\left(\mathfrak{B}_{1} \times \cdots \times \mathfrak{B}_{n}\right)$ is convolvable if $\mu \star \nu$ exists for every $\nu \in$ $\mathcal{F}_{n}\left(\mathfrak{B}_{1} \times \cdots \times \mathfrak{B}_{n}\right)$. Every $\mathcal{F}_{2}$-measure on $\mathfrak{B}_{1} \times \mathfrak{B}_{2}$ is convolvable (see the remark following Theorem 5.1; cf. [GS1]). By contrast, there exist $\mathcal{F}_{3}$-measures on $\mathfrak{B}_{1} \times$ $\mathfrak{B}_{2} \times \mathfrak{B}_{3}$ which are not convolvable (this was proved first in [GS2]; an alternate proof can be obtained by checking that if $n=2, m=1$, and $U=\{(1),(1)\}$, then there exists $\varphi \in l^{\infty}(\mathbb{N})$ such that $\lambda_{\varphi}$ given by (7.1) is not convolvable).

Projective boundedness is sharper than convolvability. If $\|\mu\|_{p b_{n}}<\infty$, then $\mu \star \nu$ exists for every $\nu \in \mathcal{F}_{n}\left(\mathfrak{B}_{1} \times \cdots \times \mathfrak{B}_{n}\right)$ (Proposition 4.1). However, the converse is false: every $\mathcal{F}$-measure given by (7.10) is convolvable, but not every such $\mathcal{F}$-measure is projectively bounded.

If $\mathcal{F}_{n}$-measures $\mu$ and $\nu$ on $\mathfrak{B}_{1} \times \cdots \times \mathfrak{B}_{n}$ are completely bounded, then $\mu \star \nu$ exists and is also completely bounded ([Y2], [ZS]). It is unknown whether completely bounded $\mathcal{F}$-measures are necessarily convolvable.

\section{Appendix}

The elementary proof in subsection i, below, of Theorem 2.5(i) uses an idea of [B4]. The proof in subsection ii of Theorem 2.5(ii) uses the main idea of the Pietsch factorization theorem [Pie] (see [LP, Proposition 3.2]). In subsection iii, we note that the factorization theorem implies the inequality. That these two ("bilinear") assertions are equivalent, in the sense that each is derivable from the other, does not extend to higher dimensions (e.g., see section 10.ii).

i. A proof of Theorem 2.5(i). Fix a one-one correspondence between $\mathbb{N}$ and $\bigcup\left\{\mathbb{N}^{j}: j=2,3, \ldots\right\}$, denoted by $n \leftrightarrow\left(n_{1}, \ldots, n_{j}\right)$, where $n \in \mathbb{N}$ and $\left(n_{1}, \ldots, n_{j}\right) \in$ $\bigcup\left\{\mathbb{N}^{j}: j=2,3, \ldots\right\}$. If $\mathbf{x}=\{\mathbf{x}(n): n \in \mathbb{N}\}$ is a scalar sequence then $\phi \mathbf{x}$ will denote the sequence given by

$$
(\varphi \mathbf{x})_{n}=\mathbf{x}\left(n_{1}\right) \cdots \mathbf{x}\left(n_{j}\right) /(j !)^{1 / 2}, \quad n \leftrightarrow\left(n_{1}, \ldots, n_{j}\right) .
$$


Observe that if $\mathbf{x} \in l^{2}$ and $\mathbf{y} \in l^{2}$, then

$$
\begin{aligned}
\langle\phi \mathbf{x}, \phi \mathbf{y}\rangle & =\sum_{j=2}^{\infty} \frac{1}{j !} \sum_{\left(n_{1}, \ldots, n_{j}\right) \in \mathbb{N}^{j}} \mathbf{x}\left(n_{1}\right) \cdots \mathbf{x}\left(n_{j}\right) \mathbf{y}\left(n_{1}\right) \cdots \mathbf{y}\left(n_{j}\right) \\
& =\sum_{j=2}^{\infty}\langle\mathbf{x}, \mathbf{y}\rangle^{j} / j !=e^{\langle\mathbf{x}, \mathbf{y}\rangle}-\langle\mathbf{x}, \mathbf{y}\rangle-1
\end{aligned}
$$

In particular,

$$
\|\phi \mathbf{x}\|_{2}^{2}=e^{\|\mathbf{x}\|_{2}^{2}}-\|\mathbf{x}\|_{2}^{2}-1 .
$$

Let $\theta=\phi / \sqrt{e-2}$. By (A.3), $\theta$ maps the unit sphere of $l^{2}$ into itself. Define $\mathbf{A}(\mathbf{x}, \mathbf{y})=e^{\langle\mathbf{x}, \mathbf{y}\rangle}-1$, and then rewrite (A.2) as

$$
\langle\mathbf{x}, \mathbf{y}\rangle=\mathbf{A}(\mathbf{x}, \mathbf{y})-(e-2)\langle\theta \mathbf{x}, \theta \mathbf{y}\rangle .
$$

By iterating (A.4), we obtain

Lemma A.1. If $\|\mathbf{x}\|_{2}=\|\mathbf{y}\|_{2}=1$, then

$$
\langle\mathbf{x}, \mathbf{y}\rangle=\sum_{k=0}^{\infty}(-1)^{k}(e-2)^{k} \mathbf{A}\left(\theta^{k} \mathbf{x}, \theta^{k} \mathbf{y}\right)
$$

Lemma A.2. Let $\mathbf{f}=\left(f_{j}\right)$ and $\mathbf{g}=\left(g_{j}\right)$ be finite sequences of real-valued functions in $\mathcal{L}^{\infty}(\mathcal{X})$ and $\mathcal{L}^{\infty}(\mathcal{Y})$, respectively, such that

$$
\sum_{j}\left|f_{j}(x)\right|^{2}=1 \quad \text { and } \quad \sum_{j}\left|g_{j}(y)\right|^{2}=1,
$$

for $(x, y) \in \mathcal{X} \times \mathcal{Y}$. Then

$$
\|\mathbf{A}(\mathbf{f}, \mathbf{g})\|_{V_{2}(\mathcal{X} \times \mathcal{Y})} \leq e+1 .
$$

Proof. Let $\left\{Z_{j}: j \in \mathbb{N}\right\}$ be a sequence of independent standard normal variables. If $Z$ is a standard normal random variable, then $\mathbf{E} e^{i s Z}=e^{-s^{2} / 2}, s \in \mathbb{R}$. Therefore, (A.6) implies

$$
e \mathbf{E} e^{i \sum f_{j} Z_{j}} e^{-i \sum g_{j} Z_{j}}=e^{\langle\mathbf{f}, \mathbf{g}\rangle}=\mathbf{A}(\mathbf{f}, \mathbf{g})+1
$$

$\left(\langle\mathbf{f}, \mathbf{g}\rangle\right.$ denotes $\left.\sum_{j} f_{j} \otimes g_{j}\right)$. This, by Proposition 2.1, implies (A.7).

If $\mathbf{f}=\left(f_{j}\right)$ and $\mathbf{g}=\left(g_{j}\right)$ satisfy the hypotheses of Lemma A.2, then Lemma A.1 implies

$$
\sum_{j} f_{j} \otimes g_{j}=\langle\mathbf{f}, \mathbf{g}\rangle=\sum_{k=0}^{\infty}(-1)^{k}(e-2)^{k} \mathbf{A}\left(\theta^{k} \mathbf{f}, \theta^{k} \mathbf{g}\right) .
$$

Therefore, by Lemma A.2,

$$
\left\|\sum_{j} f_{j} \otimes g_{j}\right\|_{V_{2}} \leq \frac{e+1}{3-e} .
$$

Finally, to obtain the preceding statement under the assumption in (2.12), merely redefine $f_{j}$ and $g_{j}$ to be, respectively, $f_{j} /\left(\sum\left|f_{j}\right|^{2}\right)^{1 / 2}$ and $g_{j} /\left(\sum\left|g_{j}\right|^{2}\right)^{1 / 2}$, at the points where they do not vanish. 
ii. Theorem 2.5(i) $\Rightarrow$ Theorem 2.5(ii). Norm $\mathcal{C}_{0}(\mathcal{X}) \times \mathcal{C}_{0}(\mathcal{Y})$ by $\|(f, g)\|=$ $\max \left\{\|f\|_{\infty},\|g\|_{\infty}\right\}$. Assume without loss of generality that $\|\mu\|_{\mathcal{F}_{2}}=1$. Let $\mathcal{W}$ be the subset of $\mathcal{C}_{0}(\mathcal{X}) \times \mathcal{C}_{0}(\mathcal{Y})$ containing all $(f, g)$ which can be written as

$$
(f, g)=\left(\sum_{k \in \mathbb{N}}\left|f_{k}\right|^{2}, \sum_{k \in \mathbb{N}}\left|g_{k}\right|^{2}\right)
$$

such that

$$
\left|\int\left(\sum_{k \in \mathbb{N}} f_{k} \otimes g_{k}\right) d \mu\right| \geq \mathcal{K}_{\mathcal{G}},
$$

where the $f_{k}$ 's and $g_{k}$ 's are elements of $\mathcal{C}_{0}(\mathcal{X})$ and $\mathcal{C}_{0}(\mathcal{Y})$, respectively. Observe that $\mathcal{W}$ is convex. Next, consider the convex open set

$$
\mathcal{O}=\left\{(f, g): \max \left\{f(x), g(y): x \in \mathcal{D}_{1}, y \in \mathcal{D}_{2}\right\}<1\right\},
$$

which, by Theorem 2.5(i), is disjoint from $\mathcal{W}$. Therefore, by the Riesz representation theorem and the Hahn-Banach theorem, there exists a pair of regular Borel measures $\left(\nu_{1}, \nu_{2}\right) \in M(\mathcal{X}) \times M(\mathcal{Y})$ such that

$$
\int_{\mathcal{X}} f(x) \nu_{1}(d x)+\int_{\mathcal{Y}} g(y) \nu_{2}(d y)<1 \quad \text { for }(f, g) \in \mathcal{O},
$$

and

$$
\int_{\mathcal{X}} f(x) \nu_{1}(d x)+\int_{\mathcal{Y}} g(y) \nu_{2}(d y)>1 \quad \text { for }(f, g) \in \mathcal{W} .
$$

(A.12) implies that $\nu_{1}$ and $\nu_{2}$ are nonnegative, and that $\left\|\nu_{1}\right\|_{M}+\left\|\nu_{2}\right\|_{M} \leq 1$.

Let $f \in \mathcal{C}_{0}(\mathcal{X})$ and $g \in \mathcal{C}_{0}(\mathcal{Y})$, and assume that $\mu(f, g):=\int f \otimes g d \mu \neq 0$. Since

$$
\left(\frac{\mathcal{K}_{\mathcal{G}}|f|^{2}}{|\mu(f, g)|}, \frac{\mathcal{K}_{\mathcal{G}}|g|^{2}}{|\mu(f, g)|}\right) \in \mathcal{W}
$$

(A.13) implies

$$
\begin{aligned}
|\mu(f, g)| & \leq \mathcal{K}_{\mathcal{G}}\left(\int_{\mathcal{X}} f(x) \nu_{1}(d x)+\int_{\mathcal{Y}} g(y) \nu_{2}(d y)\right) \\
& =\mathcal{K}_{\mathcal{G}}\left(\|f\|_{L^{2}\left(\nu_{1}\right)}^{2}+\|g\|_{L^{2}\left(\nu_{2}\right)}^{2}\right) .
\end{aligned}
$$

By the homogeneity of $\mu$, for arbitrary $c>0$ and $d>0$, (A.14) implies

$$
|\mu(f, g)| \leq \mathcal{K}_{\mathcal{G}}\left(\frac{c}{d}\|f\|_{L^{2}\left(\nu_{1}\right)}^{2}+\frac{d}{c}\|g\|_{L^{2}\left(\nu_{2}\right)}^{2}\right) .
$$

In (A.15), put $c=\|g\|_{L^{2}\left(\nu_{2}\right)}$ and $d=\|f\|_{L^{2}\left(\nu_{1}\right)}$, thus obtaining

$$
|\mu(f, g)| \leq 2 \mathcal{K}_{\mathcal{G}}\|f\|_{L^{2}\left(\nu_{1}\right)}\|g\|_{L^{2}\left(\nu_{2}\right)} .
$$

Since $\nu_{1} \neq 0, \nu_{2} \neq 0$, and $\left\|\nu_{1}\right\|_{M}+\left\|\nu_{2}\right\|_{M} \leq 1$, we can replace $\nu_{1}$ and $\nu_{2}$ in (A.16) by probability measures $\nu_{1} /\left\|\nu_{1}\right\|_{M}$ and $\nu_{2} /\left\|\nu_{2}\right\|_{M}$, respectively, thus obtaining (2.14). 
iii. Theorem 2.5(ii) $\Rightarrow$ Theorem 2.5(i). It can be assumed that the $f_{j}$ 's and $g_{j}$ 's for which (2.12) holds are simple functions, and hence, that $\mathcal{X}$ and $\mathcal{Y}$ are finite sets. Let $\mu \in \mathcal{F}_{2}(\mathcal{X} \times \mathcal{Y})$ be arbitrary, and let $\nu_{1}$ and $\nu_{2}$ be the respective probability measures on $\mathcal{X}$ and $\mathcal{Y}$ for which (2.14) holds. Then,

$$
\begin{aligned}
& \left|\int\left(\sum_{j} f_{j} \otimes g_{j}\right) d \mu\right| \leq \sum_{j}\left|\int f_{j} \otimes g_{j} d \mu\right| \\
& \quad \leq \mathcal{K}_{\mathcal{G}}\|\mu\|_{\mathcal{F}_{2}} \sum_{j}\left\|f_{j}\right\|_{L^{2}\left(\nu_{1}\right)}\left\|g_{j}\right\|_{L^{2}\left(\nu_{2}\right)} \\
& \quad \leq \mathcal{K}_{\mathcal{G}}\|\mu\|_{\mathcal{F}_{2}}\left(\sum_{j}\left\|f_{j}\right\|_{L^{2}\left(\nu_{1}\right)}^{2}\right)^{1 / 2}\left(\sum_{j}\left\|g_{j}\right\|_{L^{2}\left(\nu_{2}\right)}^{2}\right)^{1 / 2} \leq \mathcal{K}_{\mathcal{G}}\|\mu\|_{\mathcal{F}_{2}}
\end{aligned}
$$

(the last inequality is a consequence of the generalized Minkowski inequality and (2.12)). We deduce (2.13) by maximizing (A.17) over $\mu \in \mathcal{F}_{2}(\mathcal{X} \times \mathcal{Y})$.

\section{REFERENCES}

[B1] R. Blei, Multi-dimensional extensions of the Grothendieck inequality and applications, Ark. Mat. 17 (1979), 51-68. MR 81b:43008

[B2] Combinatorial dimension and certain norms in harmonic analysis, Amer. J. Math. 106 (1984), 847-887. MR 86a:43009

[B3] - Fractional dimensions and bounded fractional forms, Mem. Amer. Math. Soc. $\mathbf{5 7}$ (1985), no. 331. MR 87k:26021

[B4] An elementary proof of the Grothendieck inequality, Proc. Amer. Math. Soc. 100 (1987), 58-60. MR 88d:26039

[B5] Multi-linear measure theory and the Grothendieck factorization theorem, Proc. London Math. Soc. 56 (1988), 529-546. MR 89d:28005

[B6] _ Multilinear measure theory and multiple stochastic integration, Probab. Theory Related Fields 81 (1989), 569-584. MR 90f:60106

[B7] An extension theorem concerning Fréchet measures, Canad. Math. Bull. 38 (1995), 278-285. CMP 1995:17

[BS] R. Blei and J. H. Schmerl, Combinatorial dimension of fractional Cartesian products, Proc. Amer. Math. Soc. 120 (1994), 73-77. MR 94b:05204

[CES] E. Christensen, E. G. Effros and A. M. Sinclair, Completely bounded multilinear maps and $C^{*}$ algebraic cohomology, Invent. Math. 90 (1987), 279-296. MR 89k:46084

[DU] J. Diestel and J J. Uhl, Jr., Vector Measures, Math. Surveys, vol. 15, Amer. Math. Soc., Providence, RI, 1977. MR 56:12216

[D] I. Dobrakov, On integration in Banach spaces. VIII (Polymeasures), Czechoslovak Math. J. 37 (1987), 487-506. MR 89a:46097

[F] M. Fréchet, Sur les fonctionnelles bilinéaires, Trans. Amer. Math. Soc. 16 (1915), 215-234.

[GiIS] J. E. Gilbert, T. Ito and B. M. Schreiber, Bimeasure algebras on locally compact groups, J. Funct. Anal. 64 (1985), 134-162. MR 87d:43004

[GM] C. C. Graham and O. C. McGehee, Essays in Commutative Harmonic Analysis, Grundlehren Math. Wiss., vol. 238, Springer-Verlag, New York, 1979. MR 81d:43001

[GS1] C. C. Graham and B. M. Schreiber, Bimeasure algebras on LCA groups, Pacific J. Math. 115 (1984), 91-127. MR 86a:43003

[GS2] _ Projections in spaces of bimeasures, Canad. Math. Bull. 31 (1988), 19-25. MR 89b: 43004

[GY] C. C. Graham and K. Ylinen, Classes of trimeasures: applications of harmonic analysis, Probability Measures on Groups. X. (H. Heyer, ed.), Plenum Press, New York, 1991; pp. 169-176. MR 94g:46061

[G1] A. Grothendieck, Produits tensoriels topologiques et espaces nucléaires, Mem. Amer. Math. Soc. No. 16, 1955. MR 17:763c 
[G2] _ Résumé de la théorie métrique des produits tensoriels topologiques, Bol. Soc. Mat. Saõ Paolo 8 (1956), 1-79. MR 20:1194

[KW] S. Kwapień and W. Woyczyński, Random Series and Stochastic Integrals: Single and Multiple, Birkhäuser, Basel, 1992. MR 94k:60074

[L] J. E. Littlewood, On bounded bilinear forms in an infinite number of variables, Quart. J. Math. Oxford Ser. 1 (1930), 164-174.

[LP] J. Lindenstrauss and A. Pełczyński, Absolutely summing operators in $\mathcal{L}_{p}$-spaces and their applications, Studia Math. 29 (1968), 275-326. MR 37:6743

[McT] T. R. McConnell and M. S. Taqqu, Decoupling inequalities for multilinear forms in independent symmetric random variables, Ann. Probab. 14 (1986), 943-954. MR 87k:60053

$[\mathrm{M}]$ M. Morse, Bimeasures and their integral extensions, Ann. Mat. Pura Appl. 39 (1955), 345-356. MR 17:720a

[MT] M. Morse and W. Transue, Functionals of bounded Fréchet variation, Canad. J. Math. 1 (1949),153-165. MR 10:601a

[Pie] A. Pietsch, Absolut p summierende Abbildungen in normierten Räumen, Studia Math. 28 (1967), 333-353. MR 35:7162

[Pi] G. Pisier, Factorization of Linear Operators and Geometry of Banach Spaces, CBMS 60, Amer. Math. Soc., Providence, RI, 1986. MR 88a:47020

[Pr] P. Protter, Stochastic Integration and Differential Equations, Springer Verlag, New York, 1990. MR 91i:60148

[R] W. Rudin, Fourier Analysis on Groups, Interscience, New York, 1967. MR 27:2808

[S] R. R. Smith, Completely bounded multilinear maps and Grothendieck's inequality, Bull. London Math. Soc. 20 (1988), 606-612. MR 90m:46100

[V1] N. Th. Varopoulos, Tensor algebras and harmonic analysis, Acta Math. 119 (1968), 51112. MR 39:1911

[V2] - On a problem of A. Beurling, J. Funct. Anal. 2 (1968), 24-30. MR 39:3317

[V3] - On an inequality of von Neumann and an application of the metric theory of tensor products to operator theory, J. Funct. Anal. 16 (1974), 83-100. MR 50:8116

[Y1] K. Ylinen, On vector bimeasures, Ann. Mat. Pura Appl. 117 (1978), 119-138. MR 81a:28010

[Y2] Noncommutative Fourier transforms of bounded bilinear forms and completely bounded multilinear operators, J. Funct. Anal. 79 (1988), 144-165. MR 90a:46178

[ZS] G. Zhao and B. M. Schreiber, Algebras of multilinear forms on groups, Contemp. Math. 189 (1995), 497-511. CMP 1995:17

Department of Mathematics, University of Connecticut, Storrs, Connecticut 06269

E-mail address: blei@uconnvm.uconn.edu 